\title{
MEASUREMENT OF CURRENT DENSITY FLUCTUATIONS AND AMBIPOLAR PARTICLE FLUX DUE TO MAGNETIC FLUCTUATIONS IN MST
}

by

\section{WEIMIN SHEN}

A thesis submitted in partial fulfillment of the requirement for the degree of

Doctor of Philosophy

(Physics)

at the

UNIVERSITY OF WISCONSIN-MADISON

1992 


\title{
MEASUREMENT OF CURRENT DENSITY FLUCTUATIONS AND AMBIPOLAR PARTICLE FLUX DUE TO MAGNETIC FLUCTUATIONS IN MST
}

\author{
Under the supervision of Professor Stewart C. Prager (Physics) at the \\ University of Wisconsin-Madison
}

\begin{abstract}
Studies of magnetic fluctuation induced particle transport on Reversed Field Pinch plasmas were done on the Madison Symmetric Torus. Plasma current derisity and current density fluctuations were measured using a multi-coil magnetic probes. The low frequency $(f<50 \mathrm{kHz})$ current density fluctuations are consistent with the global resistive tearing instabilities predicted by 3-D MHD simulations. At frequencies above $50 \mathrm{kHz}$, the magnetic fluctuations were detected to be localized with a radial correlation length of about $1-2 \mathrm{~cm}$. These modes are locally resonant modes since the measured dominant mode number spectra match the local safety factor $q$. The net charged particle flux induced by magnetic fluctuations was obtained
\end{abstract}


by measuring the correlation term $\left\langle\tilde{j}_{11} \widetilde{B}_{r}\right\rangle$. The result of zero net charged particle loss was obtained, meaning the flux is ambipolar. The ambipolarity of low frequency global tearing modes is satisfied through the phase relations determined by tearing instabilities. The ambipolarity of high frequency lecalized modes could be partially explained by the simple model of Waltz based on the radial average of small scale turbulence. 


\section{ACKNOWLEDGMENT}

First and foremost, I would like to express my thanks to my major advisor, Professor S. C. Prager, for his support, advise, and patience during 6 years of my graduate study. His leads and suggestions are extremely inspiring and helpful. $I$ also wish to thank Professor R. N. Dexter, who retired almost two years ago, for his original idea of diagnostics, his enthusiasm, and his hands-on advisories.

Many thanks go to Professor J. C. Sprott, Professor S. A. Hokin and Professor P. W. Terry for countless useful discussions, suggestions and help.

I am grateful to the very fine technical support I have always received from a group of the nicest persons, including T. Lovell, J. Laufenberg, and their army of student hourlies. Their work of machine maintenance and craft assistance through these 6 years are an indispensable part of this work. I. am also grateful to Glenn Fleet, Larry Smith and many other hackers for keeping the computer, the very vital part of my research work, working daily. Their patience and dedication are the source of fine memory.

I would like to thank my fellow graduate students and postdocs who have countless useful contributions toward the work of this thesis. Let me just 
name very few of them here: Dr. J. Sarff, Dr. T. D. Rempel, S. Assadi, Dr. A. Almagri and M. Stoneking. Also, E. Hains and I. H. Tan are remembered for helping me carry out some measurements on the Tokapole II tokamak. Thanks also go to Dr. Z. Chang for many useful discussions covering the theoretical aspects of this work.

My sincerest thanks go to my wife, Xin Shen, for her continuous love and support during the past 3 years.

At last, I wish to thank my parents for their selfless and hearted support from that piece of lovely land 10,000 miles away.

This work is suported by the U.S Department of Energy. 


\section{TABLE OF CONTENTS}

Abstract $\quad$ i

Acknowledgment iii

Table of Contents $\quad \mathrm{v}$

Chapter 1. Introduction 1

Chapter 2. Review of RFP Magnetic Turbulence and

$\begin{array}{ll}\text { Transport } & 6\end{array}$

2.1 Magnetic Fluctuations in RFP Plasmas and

MHD Instabilities $\quad 7$

2.1.1 Magnetic Fluctuations Caused by MHD

$\begin{array}{ll}\text { Iristabilities } & 8\end{array}$

2.1.2 Other Turbulence models for Microscopic

Magnetic Fluctuations in the RFP $\quad 15$

2.2 Plasma Transport Induced by Magnetic

$\begin{array}{ll}\text { Fluctuations } & 16\end{array}$

2.3 Ambipolarity of Magnetic Fluctuation 
Induced Particle Fluxes

2.3.1 Ambipolarity of Flux due to External

Induced Magnetic Fluctuations

2.3.2 Ambipolarity of Flux due to Plasma

Internal Normal Modes

2.4 Other Experimental Works

Chapter 3 Experimental Appratus and Current

Density Diagnostics

3.1 Madison Symmetric Torus

3.2 Diagnostics for Turbulence and

Transport Studies

3.2.1 Current Density Diagnostics

(Forked Probe)

3.2.2 Other Diagnostics for Turbulence and

Transport Studies

Chapter 4 Measurement of Plasma Current Density and Current Density Fluctuations

4.1 Equilibrium Current Density Measurement in MST

4.1.1 Parallel Current Density Measurement in MST 


\subsubsection{Current Density Measurement During}

PPOH Experiment

4.2 Current Density Fluctuation Measurement in MST

Chapter 5 Measurement of Internal Magnetic

Fluctuation and Mode Number Spectrum

75

5.1 Internal Magnetic Fluctuation in MST

5.1.1 General Characteristics of Externally

Measured Magnetic Fluctuations

5.1.2 General Characteristics of Internal

Magnetic Fluctuations

5.2 Global and Localized Magnetic Fluctuations in MST

Chapter 6 Ambipolar Particle Flux Induced by Magnetic Fluctuations

6.1 Ambipolar Particle Transport due to Magnetic Fluctuations

6.1.1 Experimental Evidence of Ambipolar Particle Flux Induced by Magnetic Fluctuations

6.2 Interpretation of Ambipolar Flux Induced by Magnetic Fluctuations 
viii

Chapter 7 Summary and Conclusions

103

Appendix 


\section{Chapter 1: Introduction}

The Reversed Field Pinch (RFP) is an attractive scheme for confining thermal plasmas for harnessing nuclear fusion energy. One of the major obstacles for the RFP confinement scheme, and for all the magnetic confinement schemes, to achieve ignition is the excessive loss of energy and particles. The losses are anomalous compared with the estimated losses based on the classical Colomb collisional mechanism. This so called "anomalous" particle and energy trausport is found to be closely related to the turbulent state of the plasmas in tokamaks and RFPs, 1,2 , particularly at the edge of these devices.

The occurrence of turbulence in confined plasmas is observed in all magnetic confinement devices, including $\operatorname{RFPs}{ }^{3,4}$ The plasma turbulent state is marked by stochastic fluctuations of important plasma quantities, such as density, temperature, and electromagnetic fields. The fluctuations of these plasma quantities, and the correlations among them, are postulated as a major candidate for the observed anomalous plasma transport. The origin of plasma turbulence, as well as the exact physics mechanisms that relate turbulence and transport fluxes, remains one of the foremost tasks facing the 
nuclear fusion program.

Many works have been done in measuring particle anc̉ energy flux due to the fluctuations in the edge plasmas in RFP machines, ${ }^{5,6,7}$ and on tokamaks as well. 8,9 Most of the measurements detect electrostatic-fluctuationinduced-transport. The reason for this is two-fold: 1). tokamaks usually have magnetic fluctuations presumed to be too small to be meaningful source of transport; 2). quantities related to magnetic fluctuations, such as the current density fluctuations, are difficult to measure experimentally.

This thesis consists of two major parts. First, we introduce a method for measuring plasma current density using multi-coil magnetic probes. The measurement of both equilibrium and fluctuating current density will be presented. Second, we present the results of measuring the anomalous particle flux induced by magnetic fluctuations in the RFP plasmas. The ambipolarity of such flux is discussed in detail.

The measurement of particle and energy transport due to magnetic fluctuations is very important to the understanding of RFP plasma confinement. Magnetic field fluctuations are much higher $\left(\frac{\widetilde{B}}{B} \sim 1-2 \%\right)$ for RFP plasmas than for tokamak plasmas $\left(\frac{\widetilde{B}}{B} \sim 0.01 \%\right)$. The poor confinement of RFP plarmas is evidenced by the high value of the one-turn external loop 
voltage, which is believed closely related with the high value of magnetic fluctuations. 10,11 Another important impact. of magnetic fluctuations on the RFP is that the dynamo effect could be the direct result of magnetic fluctuations. The dynamo effect is a process of spontaneously generating reversed field state.

Our results show ${ }^{12}$ three important aspects of the magnetic fluctuations and transport for RFP plasmas in MST. First, we demonstrate that the magnetic fluctuations consist of two very different types of modes. The high frequency $(f>50 \mathrm{kHz})$ magnetic fluctuations are small-scale localized modes. The radial coherence lengths for those high-frequency modes are very short, giving the evidence of locally resonant modes. The wavenumber spectrum $k_{(r)}$ of these modes is a strong function of plasma radius. This is very different from the low frequency $(f<50 \mathrm{kHz})$ case in which the fluctuations are believed to be global resistive tearing instabilities. Resistive interchange modes are presumed to be the source of small-scale, highfrequency magnetic fluctuations, although the definitive conclusion on the sources of small scale magnetic fluctuation is beyond this thesis work. Second, we have measured plasma current density and current density fluctuations using a multi-coil magnetic probe from which $\nabla \times \mathrm{B}$ is obtained. 
The measured current density fluctuations at low frequency agree in many aspects with the predictiono of a 3-D MHD simulation. Third, from the zurrent density fluctuations we have calculated the net charged particle flux induced by magnetic fluctuations in the RFP. The flux is shown to be intrinsically ambipolar. The dependence of the ambipolarity of the flux on the radial electric field is discussed.

This thesis is organized as follows: Chapter 2 describes the status of theoretical and experimental study of magnetic fluctuations and fluctuationinduced anomalous transport in RFHs. Chapter 3 gives descriptions of experimental apparatus including the MST and current, density diagnostics. Chapter 4 provides the results of equilibrium and fluctuating current density measurements. Chapter 5 presents the measurements of internal magnetic fluctuations. Chapter 6 presents the results of measuring magneticfluctuation-induced particle transport. Chapter 7 gives conclusions and summaries of the works stated above. The mathematical scheme used in the statistical analysis is briefly presented in the Appendix. 


\section{REFERENCES:}

1 A. J. Wootten et, al., Physics of Fluids, B2, (1990), 2879.

$2 \quad$ P. C. Liewer, Nuclear Fusion, 25, (1985), 543.

3 I. H. Hutchingsor: 价 al., Nuclear Fusion, 24, (1984), 59.

$4 \quad$ R. J. Haye et. al., Physics of Fluids, 27, (1984), 2576.

$5 \quad$ T. D. Rempel et. al., Physical Review Letters, 67, (1991), 1438.

6 J. C. Ingraham et. al., Physics of Fluids, B2, (1990), 143.

7 H. Ji et. al., Physical Review Letters, 67, (1991), 62.

8 S. J. Zweben, et. al., Jounal of Nuclear Materials, $111 \& \mathbf{1 1 2}$ (1982), 39.

$9 \quad$ Ch. P. Ritz, et. al., Physical Review Letters, 62, (1989), 1844.

10) H. Y. W. Tsui, Nuclear Fusion, 28, (1988), 1543.

11 R. W. Moses et. al., Physics of Fluids, 31, (1988), 3152.

12 W. Shen et. al., Physical Review Letters, 68, (1992), 1319. 
Chapter 2: Review of RFP Magnetic Turbulence and Transport

The method of RFP plasma confinement ${ }^{1}$, proposed by several investigators in the initial period of fusion research, makes use of the pinch effert: the self-constriction that occurs in a plasma as a result of the passage of a unidirectional current. In RFP plasmas the mean magnetic field is mainly determined by the induction from tre plasma current itself. This is significantly different from the tokamak plasma confinement method where the mean magnetic field is determined mainly by a strong externally applied axial magnetic field. The difference in magnetic field configuration between KFP and tokamak plasmas has resulted in a profound difference, yet similar in some degree, in the stability of plasmas, plasma turbulence, and thus probably difference in anomalous plasma transport from the core to the edge. The work of this thesis is concentrated on the measurement of internal magnetic fluctuations, measurement of current density and current density fluctuations, and the measurement of non-ambipolar particle flux induced by the magnetic fluctuations. This chapter is devoted to the review of the RFP magnetic configuration, instabilities and transport mechanisms rooted in magnetic turbulence in the RFP. Special interest is paid to the particle 
transport. In Section 2.1 we give a brief review of the MHD stability and unstable modes in the RFP configuration. Section 2.2 describes the particle flux induced by magnetic fluctuations in confinement devices. Section 2.3 gives more detailed derivations and proofs of ambipolarity of particle flux induced by magnetic fluctuations.

\subsection{Magnetic Fluctuations in RFP Plasmas and MHD Instabilities}

The RFP is a toroidal axisymmetric magnetic configuration in which the plasma is confined by a combination of poloidal field $B_{\theta}$, produced by a toroidal current in the plasrna, and a toroidal field $B_{\phi}$. As shown in Fig. 2.1. $B_{\phi}$ is partly applied from external coils that surround the minor diameter of the torus, and partly generated, presumally, by some non-linear dynamic process in the plasma. The mean values of the poloidal and toroidal fields are comparable in magnitude, that is: $\left|\frac{B_{\theta}}{B_{\phi}}\right| \sim 1$. One of the features that distinguishes the RFP from the tokamak is the toroidal field. In the RFP $B_{\phi}$ is much weaker and reverses its direction on the outside of the plasma column. This reversal of field at the edge occurs automatically, under certain conditions, through the so called "dynamo" process. The term "dynamo" that 


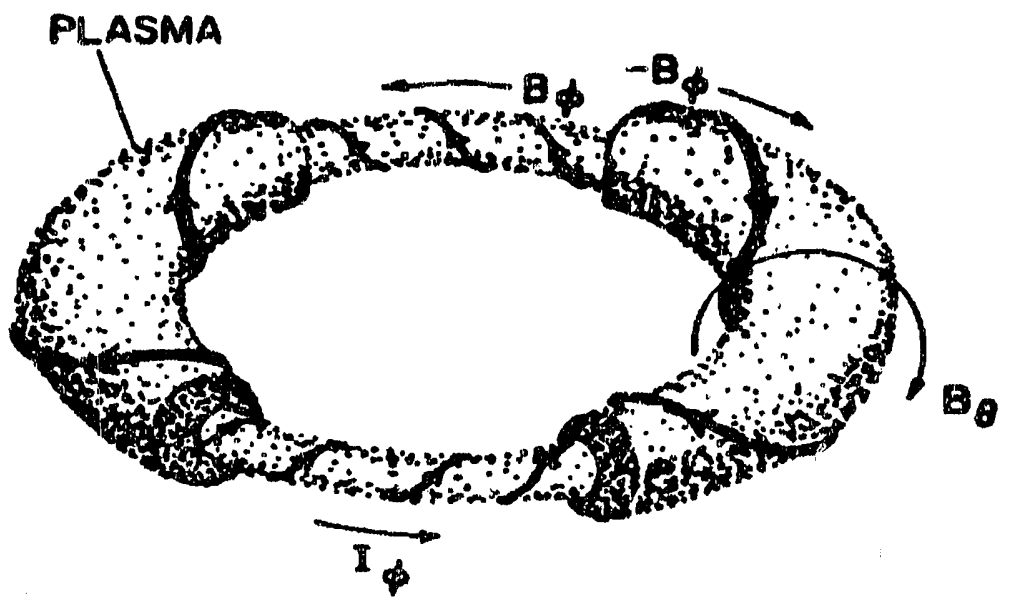

Fig. 2.1 The schematic illustration of RFP magnetic confuguration. 
describes the self generation of a mean field by a turbulent magnetic field, is borrowed from space plasma physics.

The occurrence of low frequency, large scale $\left(k_{\perp} a \sim 1\right.$ or $\left.n \sim 1\right)$ magnetic fluctuations, along with high frequency, high mode number, small scale broad-band ( $k_{\perp} a>1$ or $n>1$ ) magnetic turbulence, is commonly observed in RFP experiments. Here, $a$ is the minor radius of the plasma column, $k_{\perp}$ is the perpendicular wave number, and $n$ is the toroidal mode number, defin.ed as $n=k_{t} a . k_{t}$ here is the wave number in the toroidal direction. The niacroscopic magnetic fluctuations, larger than their counterparts in tokamaks by 2 orders of magnitude, can be adequately described by magnetohydrodynamics (MHD). The origin of microscopic magnetic turbulence, however, is not fully understocd. There are various proposed theoretical models including resistive interchange instabilities, small scale MHD instabilities, electron drift wave turbulence, etc.

\subsubsection{Magnetic Fluctuations Caused by MHD Instabilities}

From the ideal (zero resistivity) MHD point of view, the RFP is a favorable configuration against ideal instablities. The RFP operates above 
the Kruskal-Shafranov limit and obtains MHD stability through wall and shear stabilization of the plasma column.

The Suydam criterion ${ }^{2}$ states that the high value of magnetic shear has a stabilizing effect against localized pressure driven instabilities. The magnetic shear is defined as $\left(\frac{1}{P}\right)\left(\frac{d P}{d r}\right)$, where $P=r \frac{B_{\phi}}{B_{\theta}}$ is the pitch. The RFP has the advantage of possessing high shear throughout the plasma column compared to tokamak plasmas. The high magnetic shear localizes the pressure driven modes. A stability at relatively high $\beta(10-30 \%)$ is thus possible. Here $\beta$ is the ratio of plasma pressure $p$, and the total magnetic pressure in the plasma, $\beta=\frac{8 \pi p}{B^{2}}$.

The general criterion for stabilizing current driven instabilities is obtained by satisfying $\frac{d q}{d r} \neq 0$ (no pitch minimum) throughout the plasma minor radius in the RFP. The $m=1$ long-wavelength current driven (kink) instabilities, in particular, are stabilized by a conducting shell surrounding the plasma column. The effect of the shell is to provide an induced wall current that adds magnetic pressure to the plasma column. The conducting shell also exerts a limit on the field reversal parameter by the pinch stability condition..$^{3}$

The resistive magnetohydrodynamics activity, on the other hand, with its 
large growth rate, can cause substantial magnetic field fluctuations and turbulence. Furthermore, the non-linear interaction of resistive MHD modes, resulting from magnetic island overlapp between modes of different resonant surfaces, is believed to be responsible for sustainment of the RFP configuration. The non-linear coupling of modes could cause magnetic flux surfaces to break-up, thus limiting the confinement. The study of MHD instabilities in the RFP is very important to the understanding of RFP confinc ant and transport, as well as confinement and transport in general.

Three types of linear instabilities have been obtained theoretically from MHD equations under the RFP mean field configuration. These equations include the effect of finite resistivity, compressibility, and viscosity. The instabilities are identified as: TEARING modes driven by a parallel current density gradient, RESISTIVE INTERCHANGE modes (g-modes) driven by a pressure gradient, and RIPPLING modes driven by a gradient in the resistivity. It is wideiy believed that resistive tearing and perhaps interchange instabilities drive the magnetic fluctuations in the RFP. The contribution of rippling modes may not be important for RFPs Here we will comment on the theoretical and experimental aspects of tearing and interchange modes, respectively. 
In the RFP, where the safety factor $q_{(r)}$ decreases monotonically in radius, a spectrum of $m=1$ tearing modes with $n<20$ and resonant surfaces inside the reversal surface is destabilized by the resistive diffusion of the magnetic configuration away from its minimum energy state. The diffussion results in distortion of the magnetic field and the formation of magnetic islands near the resonant surfaces of each individual mode. The resonant surfaces are defined as the surfaces of a torus with $r=r_{c}$, such that $q_{\left(r_{c}\right)}=\frac{m}{n}$ and $m, n$ are integers. On those resonant surfaces, a magnetic field line, leaving at any point and traveling along the direction of the field line, will return to the original point after circling the short circumference $m$ times and the long circumference $n$ times. An infinite number of such trajectories of field lines maps out the resonant surfase at $r=r_{c^{\circ}}$. We define the modes as internally resonant if $\frac{m}{n}$ is positive, meaning the mode resonant surface is between the core and the reversal surface $r_{s}$. The modes are externally resonant when $\frac{m}{n}$ is negative, meaning the mode resonant surface is outside of $r_{s}$.

The importance of both internal and external $m=1$ tearing modes is discussed by $\mathrm{Ho}_{0}$ and Prager ${ }^{4}$. Magnetic fluctuations with very large amplitudes $\left(\frac{\widetilde{B}}{B} \sim 1-2 \%\right)$ and low frequency $(f<50 \mathrm{kHz}$ in the MST $)$ are observed in many experiments. The characteristics of those fluctuations are 
in good agreement with the theoretical predictions of $m=1$ non-linear tearing instabilities. These modes dominate the fluctuation spectrum and have global spatial structure.

Unlike the tokamak, where orily one or two linearly unstable tearing modes exist, the RFP is likely to have many unstable $m=1, n>1$ modes with their resonant surfaces inside the reversal surface. Because of the large number of unstable modes and the close spatial proximity of their resonant surfaces, strong nonlinear interactions among those modes dominate the evolution of the fluctuation spectrum. As the quasi-steady state of turbulence is reached, the $n$-spectrum is broadened with an increase of power at high $n$. The spectral broadening, which is caused by nonlinear coupling through the process of magnetic island overlap, extends the radial structure of instabilities, causing global distortion of the magnetic field configuration. This process also redistributes magnetic energy to higher $n$ modes through $m$ $=0$ modes. The physical consequence of nonlinear coupling is the formation of a large region of stochastic magnetic field and the destruction of flux surfaces between the plasma core and $r_{s}$.

The nonlinear MHD process of $m=1$ modes has been well developed computationally. It is shown from a 3-D nonlinear MHD code $\mathrm{e}^{5}$ that there 
existes a region near the core of the plasma column where magnetic flux surfaces become stochastic. The stochastic core is produced by $m=1$ magnetic island overlap. The radial extent of this re rion is dependent on the pinch parameter $\Theta\left(\Theta=\frac{B_{p}(a)}{\left\langle B_{t}\right\rangle}\right)$.

Resistive interchange modes may significantly affect confinement in RFPs. ${ }^{6,7}$ Unlike their ideal counterparts, they can be excited even in the presence of strong shear due to unfavorable average curvature of the RFP configuration. The linear growth rates of resistive interchange modes are larger than the linear growth rate of the tearing modes in high $\beta$ plasmas. Resistive g-modes are believed to be localized at their resonant surfaces with small radial coherence length $\lambda_{r}$. RFP plasnas appear to have flat pressure profiles with gradients largest near the edge. To maintain resonance with the equilibrium magnetic field, the resistive g-mode fluctuations, or any localized turbulence modes, at the plasma edge would be expected to have larger $n$, and the value of $n$ would have different signs on each side of the reversal surface. The experimental evidence of resistive interchange modes is scarse. The evidence of edge-localized magnetic turbulence and their mode number spectra could be a great help in identifying resistive g-modes in RFPs. According to one of the plasma turbulence models based on resistive- 
pressure-driven modes $^{8}$, there exist two different turbulence scale lengths: the magnetic fluctuation correlation length and the characteristic scale ler.gth for electrostatic fluctuations $\left(L_{\tilde{B}} \neq L_{\tilde{T}}\right)$. This can have important experimental implications in identifying the occurence of resistive interchange modes.

2.1.2 Other Turbulence Models for Microscopic Magnetic Fluctuations in the $R F P$

The origin of localized microscopic magnetic fluctuations $\left(k_{\perp} a \gg \vec{i}\right.$, or $n \gg$ 1 for RFP ) can also be attributed to other plasma physics mechanisms besides pressure-driven MHD instabilities.

Drift wave instabilities are considered one of the most likely causes of the observed microturbulence and anomalous transport in tokamaks. It can also play an important role in causing the observed localized broad-band magnetic turbulence in RFP plasmas since the experimental turbulence frequencies and wavenumbers on RFP devices are in the proper range for the drift wave model. Waltz has shown that ${ }^{9}$ the magnetic contributions to the drift wave turbulence and anomalous transport become more important 
as the plasma $\beta$ increases. Thus drift wave turbulence in the RFP is likely to become more significant compared with that in tokamak plasmas since RFP plasmas in general have higher $\beta$. Theoretical work on drift instabilities in the RFP at high $\beta$ has progressed substantially since the early work of theta-pinch experiments. ${ }^{10}$

Another possible candidate for the measured small-scale magnetic fluctuations is the high $\beta$ branch of the micrctearing instability. Some recent theoritical work ${ }^{11}$ based on the mixing length analysis and the variational calculation of the renormalized eigenmode equations has hinted at the importance of these modes. It is shown that the magnetic fluctuation saturation level and radial correlation length for such fluctuations is very closely dependent on temperature and terperature scale length. This is because the expression for $\frac{\delta B_{r}}{B_{0}}$ is a strong function of the temperature gradient diamagnetic frequency $\omega_{r}^{*}$. The definition of $\omega_{r}^{*}$ will be given in 2.3.1.

\subsection{Plasma Transport Induced By Magnetic Fluctuations}

Since both global and localized magnetic fluctuations can cause 
significant distortion of flux surfaces, they may limit the plasma confinement by transporting plasma particle and energy from the hot core to the cold edge region. This is because the charged particles move much faster along the magnetic field lines than across these lines. Due to their large radial extent and comparatively high fluctuation level, global $m=1$ tearing modes could drive significant anomalous particle and energy losses. In RFP the equilibrium profile of plasma and magnetic turbulence can be closely coupled. The spatial profiles of various plasma quantities govern the existence and growth rates of fluctuations through MHD stability conditions. Those MHD instabilities and magnetic turbulence in turn affect and modify the equilibrium profile through plasma transport processes.

Small fluctuations in the electric and magnetic fields lead to small fluctuations in the particle's velocities and radial positions. This can lead to transport of both particle anc energy across the confining magnetic field. In the low frequency limit ( $\omega \ll \omega_{c i}$ ), the particle's radial velocity has the form:

$$
\tilde{V}_{r}=\frac{c \widetilde{E}_{t}}{B}+\frac{V_{11} \widetilde{B}_{r}}{B}
$$

where $\widetilde{E}_{t}$ is the fluctuating electric field normal to $B, V_{/ /}$is the velocity of the 
particles along the unperturbed magnetic field, $\tilde{B}_{r}$ is the radial component of the fluctuating magnetic field, and $B$ is the equilibrium magnetic field strength.

From (2.1), we obtain the particle flux due to the fluctuating field that is a function of the correlation between two fluctuating quantities. Then the fluxes $<\tilde{n} \tilde{V}_{r}>$ from the fluctuating fields and fluctuating radial velocity for electrons and ions are respectively:

$$
\begin{gathered}
\Gamma_{e}=\frac{c\left\langle\tilde{n}_{e} \tilde{E}_{t}\right\rangle}{B}-\frac{\left\langle\tilde{j}_{e / /} \tilde{B}_{r}\right\rangle}{e B} \\
\Gamma_{i}=\frac{c\left\langle\tilde{n}_{i} \tilde{E}_{t}\right\rangle}{B}+\frac{\left\langle\tilde{j}_{i \prime \prime} \tilde{B}_{r}\right\rangle}{e B}
\end{gathered}
$$

$\Gamma_{e}$ and $\Gamma_{i}$ are the electron and ion fluxes, respectively, $j_{e / /}$ and $j_{i / /}$ are the parallel current densities from electrons and ions, defined as $j_{e^{\prime /}}=-n q v_{e / /}, j_{i / /}=$ $n q v_{i, r}$, and $<>$ represents an ensemble average.

In equation (2.2) and (2.3), we see that the first terms on the right hand side represent the particle fluxes from the electrostatic fluctuations due to $\overrightarrow{\boldsymbol{E}}$ $\times \vec{B}$ motion. The second terms involve magnetic-fluctuation-induced particle transport. This thesis will focuse on the second term and will report some 
experimental measurements of this term for RFP plasmas in the MST.

The transport mechanism of the second terms of (2.2) and (2.3) are sometimes called transport by magnetic "flutter." 12 When radial magnetic fluctuations $\widetilde{B}_{r}$ are present, the field line trajectories will deviate from the original flux surfaces that confine the plasma. As the result of such deviations, each electron now has a radial velocity perturbation given by $\tilde{v}_{r}$ $=v_{l i} \frac{\tilde{B}_{r}}{B^{\prime}} \cdot$ A portion of the rapid electron motion along the field lines is converted into radial motion. When magnetic fluctuations centered at different radii or rational surfaces begin to overlap, a braiding of the field lines begins. If the overlap is large enough that the magnetic flux surfaces are essentially destroyed, then each field line trajectory wanders stochastically away from its original location as it winds around the torus. The electrons following these wandering field lines leave the plasma with large speed. The radial transport can thus be enhanced.

Although (2.2) and (2.3) are widely used in interpreting anomalous particle transport with magnetic "flutter", the equations themselves are more general expressions. They are valid even without the presence of stochastic magnetic flux surfaces.

We also like to point out that the numerators of the second terms in (2.2) 
and (2.3) correspond to the magnetic tensor terms that in turn may drive the transverse flow. The plasma transverse flow is observerd in many tokamaks and RFPs, including MST.

The flow equation driven by turbulence is written as:

$$
\rho_{0} \frac{\partial<\boldsymbol{v} \geq}{\partial t}+\mu_{i} \rho_{0}<v>\frac{B_{0}^{2}}{B}=\frac{j \times B}{c}
$$

Here $\rho_{0}$ is the mass density, and $\mu_{i}$ is the neoclassical viscous damping frequency. The transverse component of equaiion (2.4) is (in slab geometry):

$$
\rho_{0} \frac{\left.\partial<v_{y}\right\rangle}{\partial t}+\mu_{i} \rho_{0}<v_{y}>\frac{B_{0}^{2}}{B_{y}}=-\frac{\left\langle\tilde{j}_{x} \tilde{B}_{z}\right\rangle}{c}+\frac{\left\langle\tilde{j}_{z} \widetilde{B}_{x}\right\rangle}{c}
$$

Note that the last term has the form of $\left\langle\widetilde{j}_{\|} \breve{B}_{r}>\right.$ if the parallel direction is $z$ direction and $x$ is the radial direction.

It has been demonstrated by some theoretical work ${ }^{13}$ that, taking the $j_{x}$ being from convective flux, the first term on the right hand side of $(2.5)$ is connected with the Reynolds stress tensor, $\left\langle\tilde{v}_{x} \tilde{v}_{y}\right\rangle$, with the general relation: 


$$
\frac{\partial<v_{y}>}{\partial t}=-\left\langle\frac{\left.\partial<\tilde{v}_{x} \tilde{v}_{y}\right\rangle}{\partial x}>\right.
$$

in generating transverse flow. By the same token, we argue that the second term on the right hand side in (2.5) is connected to the radial gradient of the magnetic stress tensor, $\left\langle\tilde{B}_{x} \tilde{B}_{y}\right\rangle$, in generating flow. This can be done simply by noticing that:

$$
\mu_{0} \tilde{j}_{z}=\frac{\partial \tilde{B}_{x}}{\partial y}-\frac{\partial \tilde{B}_{y}}{\partial x}
$$

Then the $<\widetilde{j}_{z} \widetilde{B}_{x}>$ term in (2.5) becomes:

$$
\frac{<\widetilde{j}_{z} \widetilde{B}_{x}>}{c}=-\frac{1}{\mu_{0} c}\left[\frac{\partial<\widetilde{B}_{x} \widetilde{B}_{y}>}{\partial x}+<\frac{\partial \widetilde{B}_{x}}{\partial y} \widetilde{B}_{x}>-<\frac{\partial \widetilde{B}_{x}}{\partial x} \widetilde{B}_{y}>\right]
$$

The first term in the square bracket at the right hand side is the $x y$ component of the magnetic stress tensor. It has the analogous form to the Reynolds stress $<\tilde{v}_{x} \tilde{v}_{y}>$ in $(2.6)$.

\subsection{Ambipolarity of Magnetic Fluctuation Induced Particle Fluxes}


It is obvious that the particle flux induced by electrostatic fluctuations is always ambipolar since the speed of the $\vec{E} \times \vec{B}$ motion is the same for ions and electrons. Magnetic fluctuation induced particle flux, on the other hand, is not necessarily ambipolar since the flux of each species depends on the thermal speed of that species. Magnetic fluctuation can induce net charged particle transport if $<\tilde{j}_{/ /} \widetilde{B}_{r}>=<\left(\tilde{j}_{i}-\tilde{j}_{e}\right) \widetilde{B}_{r}>\neq 0$, meaning the particle flux is non-ambipolar. Whether the flux is ambipolar will depend on the properties of magnetic turbulence.

Assuming the electrons and ions have the same temperature, $T_{e}=T_{i}$, then $v_{e} \gg v_{i}$ due to the mass dependence of the thermal speed of the particle. If there is no electric field holding these electrons back, a very large electric current could flow radially. As charge separation occurs, a radial electric field $E_{r}$ will build up. In this section, the ambipolarity of magnetic fluctuation induced flux will be discussed. Section 2.3.1 gives ambipolar condition for externally induced magnetic fluctuations, and 2.3.2 gives the condition for internal plasma fluctuations.

\subsubsection{Ambipolarity of Flux Due To Externally Induced Magnetic Fluctuation}


If the magnetic fluctuations are introduced externally, as in the case of external coil windings, we define them as externally induced fluctuations. For those fluctuations, the mode frequency and amplitudes at the edge are given, and they are independent of the plasma parameters.

Callen has given the ambipolarity condition for particle flux induced by external magnetic fluctuations. ${ }^{12} \mathrm{He}$ based his calculation on the high-modenumber, drift-wave type magnetic turbulence. The magnetic component of the drift wave is caused by the fluctuating current produced by the difference between the wave-induced perpendicular drifts of the ions and electrons. From the linearized Vlasov equation, we have:

$$
\begin{aligned}
& {\left[\frac{\partial}{\partial t}+\boldsymbol{v} \cdot \nabla+\Omega(v \times \hat{n}) \cdot \frac{\partial}{\partial \boldsymbol{v}}-C\right] \tilde{f}} \\
& =\frac{q}{M}\left[\nabla \phi \cdot \frac{\partial}{\partial v}+\frac{1}{c}\left(\boldsymbol{v} \times \boldsymbol{B}_{1}\right) \cdot \frac{\partial}{\partial \boldsymbol{v}}+\frac{1}{c} \frac{\partial \boldsymbol{A}}{\partial t} \cdot \frac{\partial}{\partial \boldsymbol{v}}\right] f_{m}
\end{aligned}
$$

where $C$ is the collision operator, $\tilde{f}$ can be decomposed into an adiabatic part plus a non-adiabatic part, $\tilde{f}=-\frac{q \tilde{\phi} f_{m}}{T}+g$, with $f_{m}=n_{e}\left(\frac{2 \pi T}{m}\right)^{3 / 2} \exp \left[-\left(\frac{\varepsilon-q \phi}{T}\right.\right.$ )], and $\varepsilon \equiv \frac{m v^{2}}{2}+q \phi$. The fluctuating fields are: $\boldsymbol{E}=-\nabla \phi-\frac{1}{c}\left(\frac{\partial \boldsymbol{A}}{\partial t}\right)$ and $\boldsymbol{B}_{1}=$ $\nabla \times \boldsymbol{A}, \hat{n}$ is the unit vector in the direction of $\boldsymbol{B}, \hat{n}=\frac{\boldsymbol{B}}{B}$, and $\Omega$ is the cyclotron 
frequency, $\Omega=\frac{e B}{m c}$.

To calculate the resonant electron distribution function in the presence of the magnetic "flutter", we take the lowest order of the drift kinetic equation (2.4). Assuming $\frac{\omega}{\Omega} \ll 1$ and that $\tilde{f}, \tilde{\phi}$ and $\widetilde{B}_{r}$ are proportional to $\exp [i(n \phi$ $m \theta-\omega t)]$, the specific form for $g$ is:

$$
\left(\frac{\partial}{\partial t}+v_{/ /} \hat{n} \cdot \nabla \cdot C\right) g=-i\left(\omega-\omega_{*}^{T}\right) \frac{q f_{n}}{T}\left[\tilde{\phi}_{(s)}-\frac{\tilde{A}_{/(s) v_{\| \prime}}}{c} J_{0}\left(\frac{k_{\perp} v_{\perp}}{\Omega}\right)\right]
$$

Here $\omega_{*}^{T}=\omega_{*}\left(\frac{d \ln f_{m}}{d \ln n}\right)$, and $\omega_{*}=k_{y}\left(\frac{c T_{e}}{e B}\right) \frac{d \ln n_{e}}{d r}$ is the diamagnetic frequency, $J_{0}$ is 0 th order Bessel Function, and $s$ is the unit length along the gyro orbit. The result in (2.10) is obtained by averaging the lowest order of (2.9) over a gyroperiod.

As stated above, the magnetic part of the drift wave is connected with $\tilde{j}_{\|}$ generated from $v_{\perp}$ of the charged particles. Taking $J_{0}\left(\frac{k_{\perp} v_{\perp}}{\Omega}\right) \sim 1$ for $\left(\frac{k_{\perp} v_{\perp}}{\Omega}\right) \sim$ $O$, and noticing that $\tilde{B}_{r}=i k_{y} \tilde{A}_{/ /}$, the relation in (2.10) becomes:

$$
\left(\frac{\partial}{\partial t}+v_{1 /} \vec{n}_{0} \cdot \nabla-C\right) g=-i\left(\omega-\omega_{*}^{T}\right) \frac{q \tilde{\phi} f_{m}}{T^{T}}-v_{\| \prime}\left(\frac{\widetilde{B}_{r}}{B}\right)\left(1-\frac{\omega}{\omega_{*}^{T}} \frac{\partial f_{m}}{\partial r}\right)
$$

For a complete derivation of (2.10) and (2.11), refer to the references by 
Callen ${ }^{12}$ and Tang et. al. ${ }^{14}$

Equation (2.11) gives an explicit expression for the perturbed distribution function due to the drift-wave turbulence. The two terms on the right hand side give the contributions from electrostatic and magnetic fluctuations, respectively. We label them as $g_{e}$ and $g_{m}$. The following discussions are focused on $g_{m}$ only.

Integrating the $\widetilde{B}_{r}$ term in (2.11) over the characteristic time, and noticing that $v_{/ /}\left(\frac{\widetilde{B}_{r}}{B}\right)=\frac{d x}{d t}$, we have $g_{m}$ as:

$$
g_{m} \cong-\sum_{\omega} e^{-i \omega t}\left(1-\frac{\omega}{\omega_{*}^{T}}\right) \frac{\partial f_{m}}{\partial r} \int_{-\infty}^{t} d t^{\prime}\left(\frac{d x^{\prime}}{d t^{\prime}}\right) \exp \left[-i(\omega+i v)\left(t-t^{\prime}\right)\right]
$$

Now we take $\frac{d x}{d t}$ as periodic in time with period $\tau_{\delta} \equiv \frac{2 \pi}{\omega_{\delta}}=\frac{2 \pi R N}{v_{\|}}$and also assume $x=x_{0}+\frac{1}{4} \Delta \sin \omega_{\delta} t$, then,

$$
g_{m} \equiv-\sum_{\omega} e^{-i \omega t}\left(1-\frac{\omega}{\omega_{*}^{T}}\right) \frac{\partial f_{m}}{\partial r}\left(\frac{1}{4} \Delta\right)\left\{\sin \omega_{\delta} t-i\left[\frac{\omega+i v}{\omega_{\delta}}\right] \cos \omega_{\delta} t\right\}
$$

Here $\Delta$ is the island width that is proportional to $\left|\frac{\tilde{B}_{r}}{B}\right|^{2}, \omega_{\delta}=\frac{v_{\|}}{N R}, v$ is the collision frequency and $C$ is taken from the Krook collisional model.

The induced particle flux is calculated from the velocity-space moment of 
the drift-kinetic equation by:

$$
\Gamma_{r}^{\delta} \equiv<\int d^{3} v\left[v_{\prime \prime}\left(\frac{\widetilde{B}_{r}}{B}\right)\right] g_{\delta}>
$$

Here $<>$ represents a spatial average over the radial extent of each mode.

Substituting $g_{\delta}$ from (2.13) into (2.14), one gets

$$
\begin{aligned}
\Gamma_{r}^{\delta} \cong- & \sum_{\omega=\omega_{m n}} \frac{1}{2}\left(\frac{1}{4} \Delta\right)^{2}(v+\gamma) \\
\mathrm{x} & n_{e}\left[\frac{d \ln n_{e}}{d r}-\frac{e}{T} \frac{d \Phi}{d r}-\frac{\omega e B}{c k_{y} T_{e}} \frac{d \ln n_{e}}{d r}\right]
\end{aligned}
$$

in which $\omega_{m n}$ are the set of rational-surface drift frequencies occurring within the plasma and $\gamma$ is the growth rate of the modes.

The ambipolarity of the particle flux from magnetic fluctuations is not reached automatically. The flux is ambipolar only when the right hand side of (2.10) becomes zero. The physical scenario of this is the electric field $\frac{e}{T} \frac{d \Phi}{d r}$ due to the charge separation balances the other two terms in square parentheses. This charge separation is caused by the unequal thermal speed of electrons and ions. Thus the condition for ambipolar diffusion is: 


$$
\frac{e}{T} \frac{d \Phi}{d r}=\left(I-\frac{\omega_{m n}}{\omega_{*}}\right) \frac{d \ln n_{e}}{d r}
$$

The relation in (2.16) can be written in the form of a force balance equation:

$$
e E_{x}=T_{e}\left(-\frac{d \ln n}{d r}\right)-\left(\frac{e B_{0}}{c}\right)\left(-\frac{\omega}{k_{\mathrm{y}}}\right)
$$

In (2.17), the first term on the right side represents the effect of the $\nabla p$ force resulting from the charged particle build up or depletion. The second term is the additional force required from the transverse rotation. The net effect of these two terms balances the force from the electric field on charged particles.

\subsubsection{Ambipolarity of Flux due to Internal Plasma Normal Modes}

If the magnetic fluctuations are not imposed externally, but from the intrinsic modes generated by plasma instabilities, the electric field criteria in (2.16) can not guarantee ambipolarity. 
The previous ambipolarity relation (2.16) can be written in the form of a dispersion relation:

$$
\omega_{e}^{*}-\omega-\omega_{E}=0
$$

with $\omega_{E}=k_{\mathrm{y}}\left(\frac{c E_{\mathrm{x}}}{B_{0}}\right)$ the electric field associated Doppler-shift frequency.

Because of the fact that for intrinsic normal modes, the mode frequencies are Doppler shifted by $\omega_{E}$, the left hand side of (2.18) becomes independent of $\omega_{E}$ and the ambipolar condition is independent of the radial electric field $E_{0}$.

R. E. Waltz ${ }^{15}$ has considered the intrinsic normal modes, under the conditions: 1) the modes are localized near their singular surfaces; 2) $\widetilde{B}_{/ /}=0$. He shows that the particle flux induced by this type of magnetic fluctuation, indeed satisfies an ambipolarity condition, independent of radial electric field. However, he did not demonstrate that the flux is pointwise ambipolar, but only that $\int\left(\Gamma_{i}-\Gamma_{e}\right) d x=0$ when integrated over the radial extent of the mode width.

Consider the kinetic equation for a distribution function $f$ of electrons, where $f=f_{m}+\tilde{f}$ with $f_{m}=n_{e}\left(\frac{2 \pi T}{m}\right)^{-3 / 2} \exp \left[-\left(\frac{E-q \Phi}{T}\right)\right]$. Here $E=\frac{1}{2} m v^{2}$. The linear Vlasov equation becomes: 


$$
\begin{gathered}
{\left[\frac{\partial}{\partial t}+v \cdot \nabla+\frac{e}{m}\left(E_{0}+\frac{v}{c} B_{0}\right) \cdot \nabla_{\mathrm{v}}\right] \tilde{f}} \\
=-\frac{e}{m}\left(E+\frac{v}{c} B\right) \cdot \nabla_{\mathrm{v}} f_{m}+[C(\tilde{f})-\langle C(\tilde{f})\rangle]
\end{gathered}
$$

where

$$
C(\tilde{f})=\frac{e}{m}\left(E_{o}+\frac{v}{c} B_{0}\right) \cdot \nabla_{\mathrm{v}} \tilde{f}
$$

and the perturbed fields are derived from

$$
\begin{aligned}
& \tilde{E}=-\nabla \tilde{\phi}+\frac{1}{c} \frac{\partial \tilde{A}}{\partial t} \\
& \tilde{B}=\nabla \times \tilde{A}
\end{aligned}
$$

The field must satisfy Poisson's and Ampere's equations,

$$
-\nabla^{2} \tilde{\phi}=4 \pi \sum \tilde{\rho}
$$




$$
-\nabla_{\perp}^{2} \tilde{A}=4 \pi \sum \tilde{j}_{\mathrm{z}}
$$

Fourier analysis in frequency $\omega$ and wavenumber $k_{\mathrm{y}}$, followed by integration over unperturbed particle orbits with constants of motion $x_{G}=x$ $+\frac{v_{y}}{\omega_{c}}$ and $\varepsilon=\frac{1}{2} m v^{2}+e \phi_{0}(x)$, and then gyrophase averaging, gives the linear response as

$$
\tilde{f}_{k}(x)=-\frac{e f_{m}}{T}\left\{\tilde{\phi}_{k}(x)-\frac{\left(\omega^{\prime}-\omega_{*}^{T}\right)}{\omega^{\prime}-k_{z} v_{z}+i \varepsilon} J_{0}^{2}\left[\tilde{\phi}_{k}(x)-\frac{v_{z}}{c} \tilde{A}_{k}(x)\right]\right\}
$$

where $\omega^{\prime}=\omega+\omega_{E}, \omega_{c}=\frac{e B_{0}}{m c}, J_{0}$ is the Bessel function, and $k_{z}=k_{y}\left(\frac{x}{L_{s}}\right)$ with $L_{s}$ the shear length for the magnetic field $B_{0}$.

The turbulent collision operator is then:

$$
\left.<C(\tilde{f})>=\frac{e}{m} \sum_{\mathrm{k}}\left(\tilde{E}_{k}^{*}(x)+\frac{v}{c} \tilde{B}_{k}^{*}(x)\right) \cdot \nabla_{v} \tilde{f}_{k^{\prime}} x\right)
$$

where $*$ represents complex conjugate.

The flux of particles induced by fluctuations is calculated by taking successive velocity moments of equation (2.25). Taking the $O$ th order momentum $\int d v(1)$ gives the particle continuity equation: 


$$
\frac{\partial n}{\partial t}+\frac{\partial \Gamma_{x}}{\partial x}=0
$$

where $n \equiv \int d v\left(f_{m}\right)$ and $\Gamma_{x}=n v_{x} \equiv \int d v\left(v_{x} f_{m}\right)$. The $\int d v\left(m v_{y} f_{m}\right)$ moment gives the $y$ momentum equation

$$
m \frac{\partial \Gamma_{y}}{\partial t}+\frac{\partial P_{x y}}{\partial x}+\frac{e \Gamma_{x}}{c} B_{O}=R_{y}
$$

where $R_{y} \equiv \int d v<C(\tilde{f})>\left(m v_{y} f_{m}\right)$ is the $y$ momentum input from the perturbed field, and $P_{x y} \equiv \int d v\left(v_{x} v_{y} f_{m}\right)$ is the stress tensor. It is easy to show that:

$$
R_{y}=\sum_{\mathrm{k}} \tilde{\rho}_{k} \tilde{E}_{y k}^{*}+\sum_{\mathrm{k}}\left(\tilde{j}_{z k} \frac{\tilde{B}_{x k}^{*}}{c}\right)
$$

where $\tilde{o}_{k} \equiv \int d v\left(e \tilde{f}_{k}\right)$ and $\tilde{j}_{z k} \equiv \int d v\left(e v_{z} \widetilde{f}_{k}\right)$.

The equation of $x$ momentum similar to (2.23) is

$$
m \frac{\partial \Gamma_{x}}{\partial t}+\frac{\partial P_{x x}}{\partial x}-e n E_{0 x}-\frac{e \Gamma_{y}}{c B_{0}}=R_{x}
$$


with

$$
R_{x}=\sum_{\mathrm{k}} \tilde{\rho}_{k} \tilde{E}_{x k}^{*}+\sum_{\mathrm{k}}\left(\tilde{j}_{z k} \frac{\widetilde{B}_{y k}^{*}}{\mathrm{c}}\right)
$$

Assuming the only time-dependent contribution to $\Gamma_{y}$ is from a timedependent $E_{0 x} \times B_{0}$ motion, and writing $(2.30)$ as

$$
m \frac{\partial \Gamma_{x}}{\partial t}-e n E_{O x} \cdot \frac{e \Gamma_{y}}{c B_{0}}=0
$$

the particle flux obtained from (2.28) using (2.32) is

$$
\Gamma_{x}=\sum_{k} \tilde{n}_{k}\left(\frac{e \tilde{E}_{y k}^{*}}{B_{0}}\right)+\sum_{\mathrm{k}}\left(\frac{\tilde{j}_{z k}}{e} \frac{\widetilde{B}_{x k}^{*}}{B_{0}}\right)+\frac{e^{2} m n}{e B_{0}^{2}} \dot{E}_{\alpha x}-\frac{c}{e B_{0}} \frac{\partial P_{x y}}{\partial x}
$$

The first term in (2.33) is the $\tilde{\boldsymbol{E}} \times \widetilde{\boldsymbol{B}}$ flow induced by the electrostatic perturbation. This term is always ambipolar. The second term is the flow induced along the perturbed magnetic field. The third term is the polarization drift that is sizable for the ions. The last term contains the effect of viscous stress.

For externally imposed magnetic pertirbations, $\widetilde{B}_{x k}$ is given and $\omega$ is 
assumed to be real. It can be shown that the condition for particle flux to be ambipolar is the same as (2.16). For externally imposed magnetic perturbations, it is possible to make the species have equal flow by adjusting $E_{0 x}$ so that the net charged particle flow is zero.

In the case of intrinsic normal modes, $\widetilde{B}_{x k}$ is not given, and $\omega$ can be a complex quantity. To solve the ambipolarity condition, one needs to go back to (2.33), and write $j$ as a function of $B$ and $E$ governed by Ampere's Law. Assuming $\widetilde{B}_{\| /}=0$, then

$$
j_{x}=-4 \pi \dot{E}_{o x}
$$

and

$$
\tilde{j}=\nabla_{\perp}^{2} \tilde{A}
$$

Then (2.26) becomes

$$
j_{x}\left[1+\left(\frac{\omega_{p e}^{2}}{\omega_{c e}^{2}}\right)\left(\frac{m_{i}}{m_{e}}\right)\right]=\sum_{k} \tilde{j}_{z k} \frac{\tilde{B}_{x k}^{*}}{B_{0}}-\left(\frac{c}{B_{0}}\right) \frac{\partial P_{x y i}}{\partial x}
$$


with $\omega_{p e}$ the electron plasma frequency defined as $\left(\frac{4 \pi n_{e} e^{2}}{m_{e}}\right)^{1 / 2}$.

Next we consider $j_{x}$ from the magnetic fluctuations only. The current will flow radially unless $\sum_{\mathrm{k}} \widetilde{j}_{z k} \widetilde{B}_{x k}^{*}=0$. The current flow is considerably reduced by the ion polarization drift since $\left(\frac{\omega_{p e}^{2}}{\omega_{c e}^{2}}\right)\left(\frac{m_{i}}{m_{e}}\right)>>1$ for typical plasma parameters as addressed by some early theoretical work ${ }^{16}$.

For local normal modes, $<\sum_{\mathrm{k}} \widetilde{j}_{z k} \widetilde{B}_{x k}^{*}>$ is zero because

$$
\begin{aligned}
& \operatorname{Re}<\tilde{j}_{z k} \tilde{B}_{x k}^{*}>\equiv \int_{-\mathrm{L}}^{\mathrm{L}} \frac{d x}{2 L} \operatorname{Re}\left(\tilde{j}_{z k} \tilde{B}_{x k}^{*}\right) \\
& =\int_{-\mathrm{L}}^{\mathrm{L}} \frac{d x}{2 L} \operatorname{Re}\left[\frac{c}{4 \pi}\left(\frac{\partial^{2}}{\partial x^{2}}-k_{y}^{2}\right) \tilde{A}_{z k} i k_{y} \tilde{A}_{z k}^{*}\right] \\
& =-\left.\frac{c}{4 \pi} k_{y} \operatorname{Im}\left(\frac{\partial}{\partial x} \tilde{A}_{z k} \tilde{A}_{z k^{\prime}}^{*}\right)\right|_{\cdot \mathrm{L}} ^{\mathrm{L}}(2 L)^{-1}=0
\end{aligned}
$$

since $\tilde{A}$ disappears at $x=L$. Here $L$ is the length that is larger than the mode radial extent.

\subsection{Other Experimental Works}

In this section, measurement of low frequency magnetic field fluctuations 
will be reviewed. Magnetic fluctuations have long been observed in stabilized pinch discharges. In early experiment on ZETA and the MK-IV torus, clear evidence of principally $m=1$ kink modes was obtained. This review includes neither of these early results, nor large sawteeth disturbances, as seen for high $\Theta$ discharges in ZT-40M at Los Alamos ${ }^{17}$. The review emphasizes the results from the later generation of machines with medium to high RFP plasma parameters. The lack of transport measurements from magnetic fluctuations presented in this review does not diminish the importance of it.

\section{OHTE}

One of the early measurements of magnetic fluctuations was made on the OHTE RFP device at General Atomics. ${ }^{18}$ The observed fluctuation spectrum had a dominant peak at $m=1, n \sim 10$. The oscillation frequency was close to the diamagnetic drift frequency. The phase velocity was opposite to the applied external electric field, that is in the direction of electron diamagnetic drift velocity for the RFP edge plasma.

The fluctuation amplitude profile was measured over the plasma minor radius. This profile was compared with a linear calculation of the internal 
resistive kink (tearing) mode profile. The measured profile was in good agreement with the dominant tearing mode profile.

The amplitude of $\widetilde{B}$ was found as a function of magnetic Reynolds number $S$. A scaling relation $\frac{\widetilde{B}}{B} \sim S^{-1 / 2}$ was obtained within the experimental uncertainties.

Magnetic fluctuation measurements were done later with the thick conducting shell removed on OHTE. ${ }^{19,20}$ MHD activities measured were similar for both the thick conducting shell and thin resistive shell. A new feature, with the modes localized toroidally, was seen on magnetic fluctuations with a thin resistive shell. This so called "slinky" mode is believed to be the result of phase locking of several internal resistive kink modes due to nonlinear mode coupling. It is postulated that the formation of these modes drives the plasma toward the Taylor relaxed state.

\section{ETA - BETA II}

Magnetic field fluctuations have been studied in the ETA-BETA II RFP device in Padova, Italy as a function of density, time and radius. ${ }^{21}$ Two sets of internal magnetic coils measuring all 3 components were inserted horizontally along the minor radius of the tor us. 
The r.m.s. values of the magnetic fluctuations at several frequency intervals versus filling density were obtained to study the variation of fluctuation versus density. Different components exhibit a similar behavior, and the amplitude of higher frequency $(f>50 \mathrm{kHz})$ fluctuations shows a rapid decay with increasing filling density, whereas the lower frequency amplitude varies only a little with density. This result also suggested that the behavior of high frequency fluctuations versus density was independent of the mean field configuration.

For all three components, the lower frequency portion exhibits a longer radial (transverse) correlation length $(>3 \mathrm{~cm}$ ) than the higher frequency portion $(\sim 1 \mathrm{~cm})$. The radial dependence of the correlation length was found to be weak.

The general features of the measured low frequency fluctuations were compared with MHD calculation. A strong similarity between the measured signal and resistive MHD modes was found in the time and space variation of the modes. Using the Rechester-Rosenbluth transport mechanism, ${ }^{22}$ the calculated energy confinement time is the same as the experimental value.

\section{HBTX-1A}


An array of edge magnetic coils has been used to measure the magnetic fluctuation structure in the HBTX-1A RFP at Culham Laboratory in the UK. ${ }^{23}$ Statistical analysis techniques have been applied to the measured data. The measured fluctuations have poloidal mode numbers dominated by 0 and 1 low frequency modes. The main peak in the $n$-spectrum is $n \sim 10$ internally resonant modes. Also seen is a large, coherent rotation of the magnetic perturbation with a rotating frequency close to the diamagnetic drift frequency. The magnetic perturbations correspond qualitatively with the expectations of resistive MHD instabilities.

The island width of the leading $m=1$ modes estimated from measured $\widetilde{B}_{r}$ is rather large $\left(\frac{\omega}{a}>30 \%\right)$, and the magnetic field lines are calculated to be stochastic due to island overlapping. The estimated energy confinement time from destroyed flux surfaces gives $\tau_{E} \sim 50 \mu s$, similar to the experimental result.

\section{TPE-1RM15}

Magnetic fluctuations on the TPE-1RM15 RFP device in the Electrotechnical Laboratory in Japan have been reported. ${ }^{24}$ The work focuses on the measurement of MHD magnetic fluctuations during dynamo activity 
and its effect on plasma energy confinement. A large number of magnetic pickup coils are positioned between the liner and the shell covering a section of the toroidal and poloidal circumferences of the torus.

It was discovered that for high $\Theta$ discharge conditions, few ( often only one or two ) $m=1$ dominant modes grow to large amplitude, until a sudden transition occurs in which the peak of the $n$ spectrum is shifted. High $n$ modes are excited. This is believed to be the result of nonlinear coupling of $m=1, \Delta n=1$ modes to generate $m=0$ toroidal flux. Low $\Theta$ discharges show qualitatively the same characteristics, such as mode growth, generation of high $n$ modes, and $n$ spectrum broadening, although differences on the number of dominant modes and time scales exist.

The effect of dominant MHD $m=1$ modes on energy confinement has been investigated. By ramping the plasma current down from some initial higher level, the discharges were kept with the same $\Theta$ and $\beta$ values, but with lower MHD activity as required by the dynamo effect. The global confinement time is improved by about a factor of 1.5. Inverse energy confinement time $\tau_{E}^{-1}$ is observed to depend on the r.m.s. value of the $m=1$ fluctuation amplitudes. The dependence of fluctuation level on Reynolds number is found to be $\frac{\widetilde{B}}{B} \sim S^{-1 / 3}$. 


\section{REFERENCES:}

$1 \quad H$. A. Bodin and A. A. Newton, Nuclear Fusion, 20, (1980), 1225.

2 B. R. Suydan, in Peaceful Uses of Atomic Energy (Proceedings of 2nd International Conference, U.N., Geneva, 1958), Vol. 31, (1958), 157.

$3 \quad$ D. C. Robinson, Plasma Physics, 13, (1971), 439.

$4 \quad$ Y. L. Ho and S. C. Prager, Physics of Fluids, 31, (1988), 1637.

$5 \quad$ D. D. Shnack, et. al., Physics of Fluids, 28, (1985), 321.

$6 \quad$ B. A. Carreras et. al., Physics of Fluids, 30, (1987), 1388.

7 J. B. Taylor and J. W. Connor, Physics of Fluids, 27, (1984), 2676.

$8 \quad$ B. A. Carreras and P. H. Diamond, Physics of Fluids B1, (1989), 1011.

$9 \quad$ R. E. Waltz, Physics of Fluids, 28, (1985), 577.

10 H. A. Bodin and A. A. Newton, Plasma Physics, 12, (1969), 2175.

11 G. G. Craddock and P. W. Terry, Physics of Fluids, B3, (1991), 3286.

12 J. D. Callen, Physical Review Letters, $\underline{3} 2$, (1977), 1549.

13 P. H. Diamond and Y.-B. Kim, Physics of Fluids, B3, (1991), 1626.

14 W. M. Tang et. al., Nuclear Fusion, 16, (1976), 191.

$15 \quad$ R. E. Waltz, Physics of Fluids, 25, (1982), 1269.

16 S. Inoue et. al., Nuclear Fusion 19, (1979), 1252. 
17 R. B. Howell et. al., Physics of Fluids, 30, (1987), 1828.

18 R. J. La Haye et. al., Physics of Fluids, 27, (1984), 2576.

19 R. J. La Haye et. al., Nuclear Fusion, 28, (1988), 918.

20 T. Tamano et. al., Physical Review Letters, 59, (1987), 1444.

21 V. Antoni and S. Ortolani, Plasma Physics, 25. (1983), 799.

22 A. B. Rechester and M. N. Rosenbluth, Physical Review Letters, 40 , (1978), 38.

23 I. H. Hutchinson et. al., Nuclear Fusion, 24, (1984), 59.

$24 \quad$ K. Hattori et. al., Physics of Fluids, B3, (1991), 3111. 
Chapter 3: Experimental Apparatus and Current Density Diagnostics

This chapter gives a brief description of the MST device, and the diagnostics used in measuring plasma current density. This diagnostic apparatus is called the "forked" probe. Emphasis is given to the unique features of both the MST device and the "forked" probe.

\subsection{Madison Symmetric Torus}

The Madison Symmetric Torus ${ }^{1}$ (MST) is a reversed field pinch device that is large in size, moderate in plasma parameters. One of the experimental goals of the MST device is to study the edge plasma physics involving turbulence and transport. This section gives a brief description of MST, interested readers can refer to Reference 1 for detail on this feature.

MST is a torus with $1.5 \mathrm{~m}$ major radius and $52 \mathrm{~cm}$ minor radius. The machine has a $5 \mathrm{~cm}$ thick aluminum shell acting as the stabilizing conducting shell, the toroidal field winding, and the vacuum chamber. The shell has two ( 1 poloidal, 1 toroidal) gaps to allow the magnetic fields to 
penetrate into the shell. Magnetic field errors are expected to be significant at the gap due to discontinuity of the image current flowing at the inner wall of the shell during the discharge. Much work on design, correction, and feedback has been and will be done to eliminate or minimize the field errors at the gaps. ${ }^{2}$

The shell also serves as the conducting boundary that is required ${ }^{3}$ for linear MHD stability of the equilibrium magnetic field configuration. Both experimental and theoretical studies have shown that the RFP plasma can not sustain its equilibrium magnetic field configuration against gross MHD instability without the close conducting boundary.

The conducting wall is also a part of the toroidal field circuit. This is done by running a current from the toroidal field power supply through the conducting shell. Doing so eliminates the usual ripple effect caused by the toroidal field windings. The elimination of toroidal field coils on the conducting shell also makes it easier for diagncstic access to the machine.

The poloidal field is provided by three field windings: continuity winding, bias winding and poloidal field winding. The continuity winding is to reduce the field distortion at the gap caused by the back-flowing surface current at the outer surface of the conducting shell. Besides the poloidal 
field winding providing the confinement field, the bias winding is to add a bias flux to the core in order to achieve the maximum flux swing of 2 VoltSeconds.

The Ohmic heating field is provided by a 2 Volt-Second iron core transformer with a 20 turn primary winding.

Fig 3.1 shows a side view of MST. Many structures mentioned above are illustrated.

On the bottom of the aluminum shell are 192 small holes for vacuum pumping. The reason for having a large number of small holes rather than a fer: large pumping holes is to minimize the field error caused by the holes. Because the conducting shell carries currents, the presence of large pumping holes would cause significant field distortion at the boundary. For the same reason there are no large diagnostic port holes on the machine. The largest holes for diagnostics are $11.4 \mathrm{~cm}$ in diameter.

MST is a machine built primarily for studying physics issues, such as plasma turbulence and transport, field error reduction, edge physics, high- $\beta$ plasma confinement, etc. It is not a device that is expected to reach fusion reactor conditions. This device is receiving great interest from the RFP research program world wide, and is an important supplement to the next 

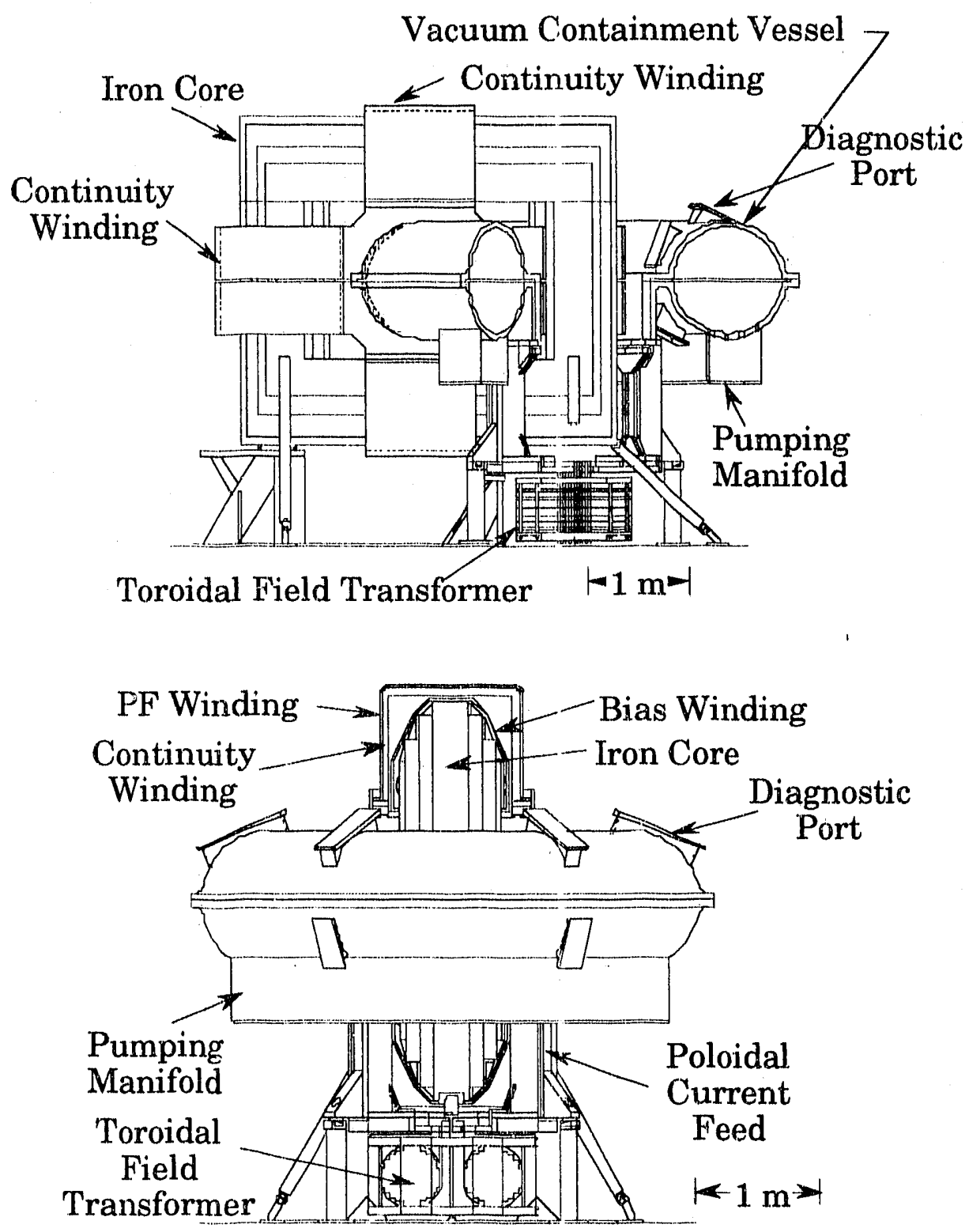

Fig. 3.1 The side views of Madison Symmetric Torus. 
generation of large size, high plasma par ameter machines such as the RFX that is beginning operation in Italy. Some key plasma parameters obtained in MST are: plasma current $Y_{p}=600 \mathrm{kA}$, electron density $n_{e}=2 \times 10^{19} \mathrm{~m}^{-3}$, electron temperature $T_{e}=400-500 \mathrm{eV}$, ion temperature $T_{i}=500 \mathrm{eV}$, average $\beta \sim 10 \%$ and confinement time $\tau_{E} \cong 1 \mathrm{~ms}$.

\subsection{Diagnostics For Turbulence and Transport Studies}

\subsubsection{Current Density Diagnostics (Forked Probe)}

Direct measurement of the plasma current density is difficult. Current density fluctuation measurement is even harder. There have been few reports of equilibrium $j$ measurement. To our knowledge, there has been no report of $\tilde{j}$ measurements at all. On the other hand, $j$ and $\tilde{j}$ are very important quantities involved in obtaining the magnetic fluctuation induced particle flux, as described by (2.2) and (2.3). This thesis describes some of the work involving current density fluctuation measurements done on the MST reversed field pinch. This section gives a brief description of the diagnostics used to measure plasma current density and current density 
fluctuations on MST. The main diagnostic apparatus is a multi-coil magnetic probe, called a "forked" probe. We focus our descriptions on the unique features of the "forked" probe that differentiates it from the conventional magnetic probe.

The "forked" probe consists of 8 magnetic pick-up coils placed in 2 stainless steel tubes with 4 coils in each one (Fig. 3.2). The tube has a $3 / 16^{\prime \prime}$ O.D. with a $0.005^{\prime \prime}$ thick wall. The tubes also act as the electrostatic shield for the probe. The magnetic pick-up coils are mounted in the tube with the help of small coil holders made from machinable ceramic that have been

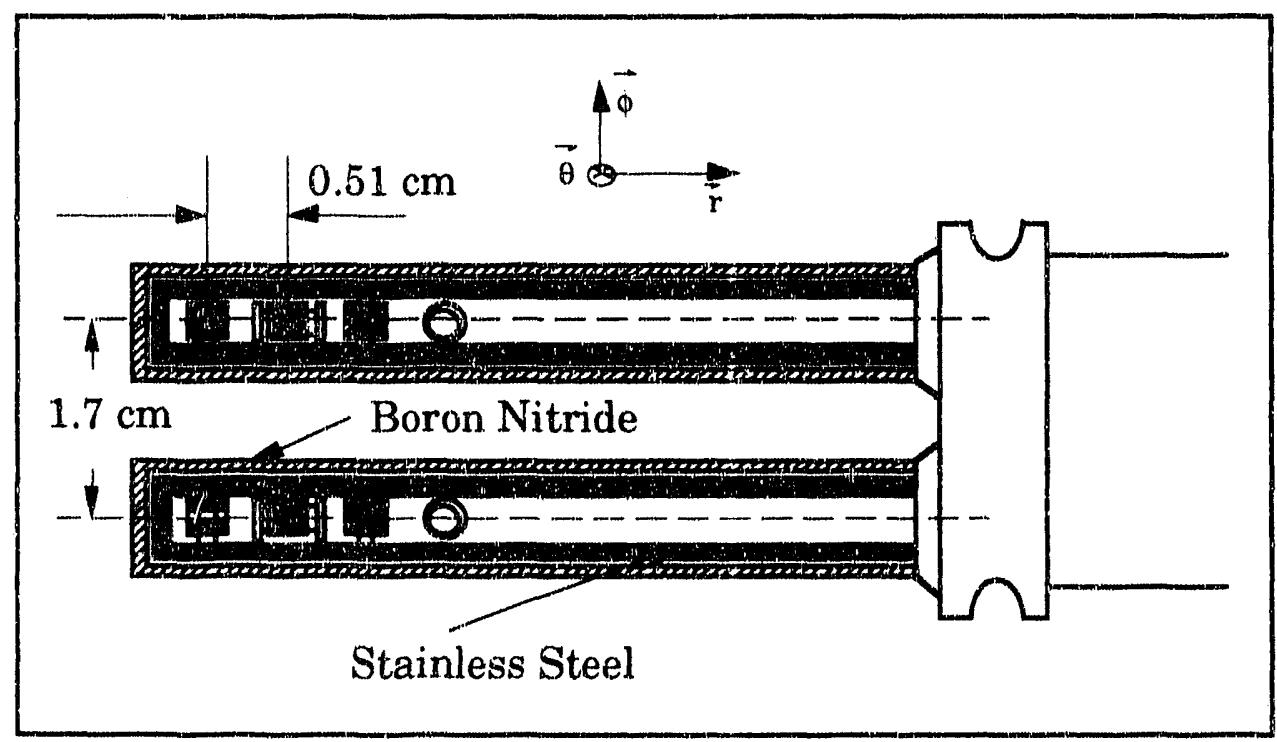

Fig. 3.2 The structuare of the "forked" probe for current density measurement. 
baked for over $10 \mathrm{hrs}$ at $2000^{\circ} \mathrm{C}$. The 2 tubes are $1.7 \mathrm{~cm}$ apart, leaving an open area in between to allow plasma current to flow. On the outside of the stainless steel tubes are Boron-Nitride tubes of 3/8" O.D. and 0.0625" thick wall. The boron-nitride tubes serve as the particle shield and heat shield of the probe.

First, let us review some of the basics of measuring magnetic field by magnetic pick-up coils. Magnetic probes usually consist of magnetic field pick-up coils, or sometimes called magnetic sensing coils. Each coil is a wire forming a closed loop. For the purpose of amplification, the coil usually has more than one turn. According to Ampere's law, the voltage at the open ends of the loop is dependent of the time rate of change of the magnetic flux encircled by the loops, that is:

$$
\frac{d \Phi}{d t}=A \frac{d B}{d t}=-V
$$

$\Phi$ is the magnetic flux, and $A$ is the total area of the coil.

When placed in a plasmas with fluctuating electromagnetic fields, a voltage signal could be obtained at a sensing coil due to the change of magnetic flux encircled by the coil. This change of flux is caused by the 
change of magnetic field $\left(\frac{d B}{d t} \neq 0\right)$. The voltage signals are then sent to electronic analog integrators. The integrators are designed such that the output signal is the analog integral of the input signals.

Fig. 3.3 is the schematic diagram of the procedure for measuring magnetic fields using pick-up coils.

The probe is usually aligned in such a way that the first and the third coils from the probe tip on each SS tube measure the toroidal field, with the open area between the two tubes facing the poloidal direction. The second coil measures radial magnetic field, and the fourth measures poloidal field.

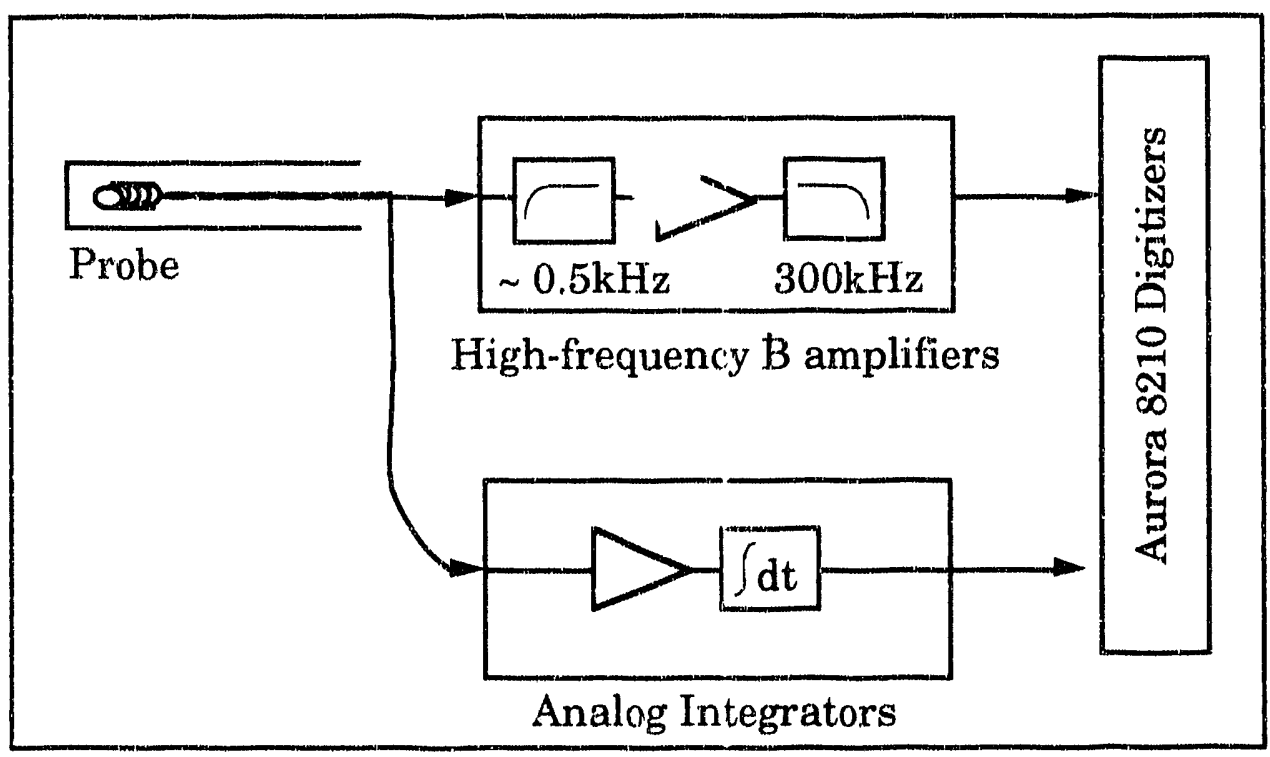

Fig. 3.3 The system configuration of data taking and analyzing used in the "forked" probe experiment. 
This alignment is for measuring poloidal current density that is close to the $\dot{j}_{/ /}$and $\tilde{j}_{/ /}$in the RFP plasma edge.

According to Ampere's law: $\nabla \times B=\mu_{0} j$. The measurement of $j$ can be obtained by measuring the spatial gradient of various components of $B$. Experimentally, this spatial gradient approach requires very high accuracy in measuring $B$ and spatial distances.

The current density can then be obtained by taking the gradient of the measured field strength within this array. Most of the previous methods implementing single cylinder type of probes have a generic fault due to the fact that there is no plasma current flowing through the coil array. The continuous flow of plasma current is blocked by the probe. The structure of this "forked" shape, in contrast, has an open space in the middle of the coil array. This open area allows the current to flow through the middle of the coil array when the probe is inserted into the plasma during the experiment.

Fig 3.4(a) shows schematically how current density measurements are done using the "forked" probe. The coils are placed at locations marked 1, 2, 3 , and 4. Coil \#1 and \#3 are on the same probe lead (same SS tube) and radially displaced, as are coils 2 and 4 . Only the coils aligned toroidally are labeled in Fig. 3.4(a). The two leads aretoroidally displaced when measuring 


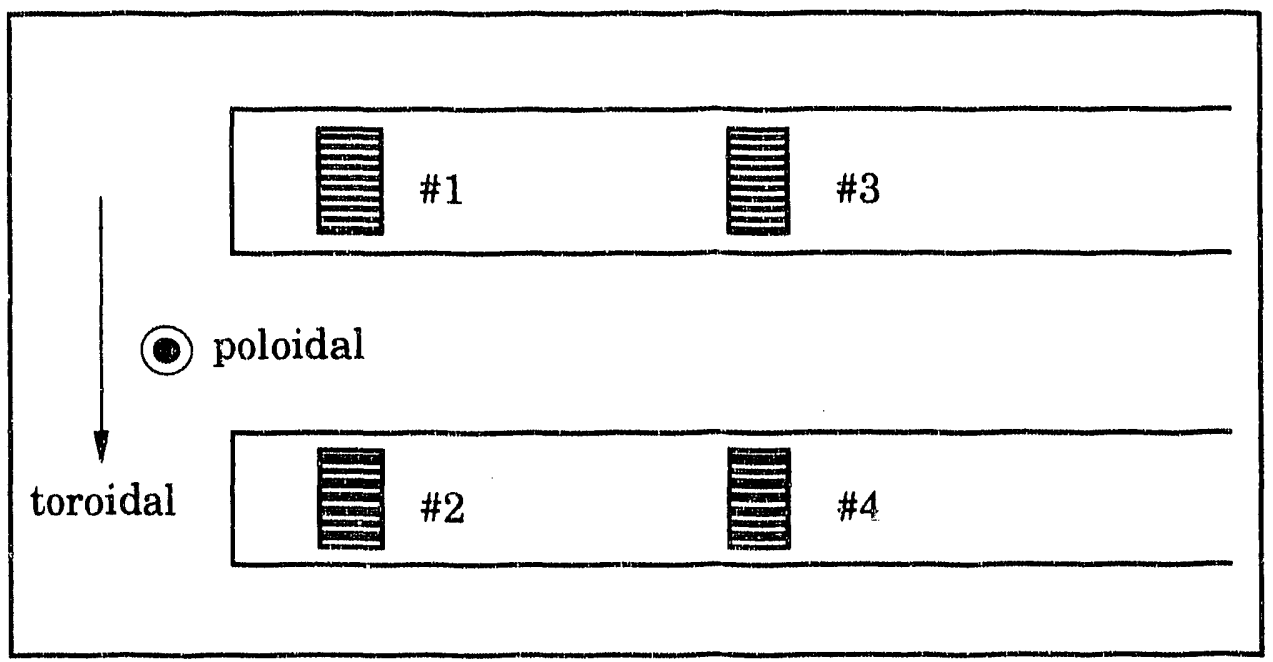

(a)

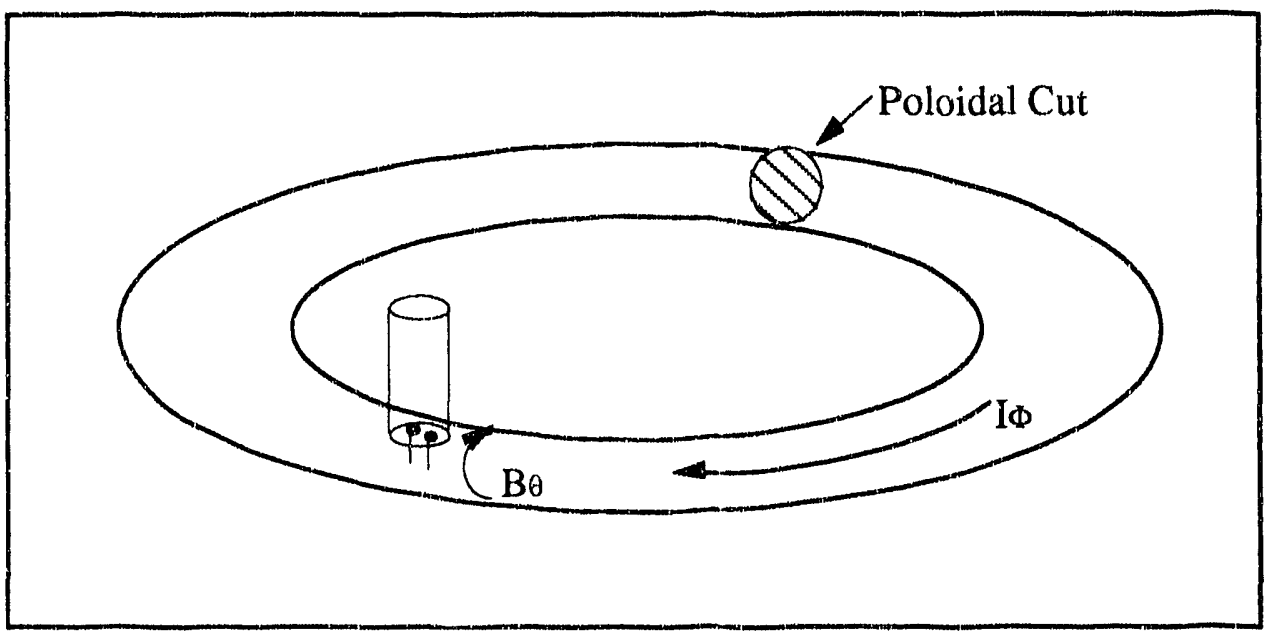

(b)

Fig. 3.4 (a) A schematic structure of "forked" probe for measuring current density; (b) The probe is inserted vertically (from the top) into the plasmas. 
poloidal current density.

The current density $j$ is obtained by measuring the gradients of B using Ampere's law: $\nabla \times \boldsymbol{B}=\mu_{0} j$. Taking the poloidal component of $j$, we have:

$$
m_{0} j_{p}=\frac{\partial B_{t}}{\partial r}-\frac{1}{R}\left(\frac{\partial B_{r}}{\partial \phi}\right)
$$

To obtain equilibrium $\mu_{0} j_{p}$, we take $\Delta B_{1}=B\left(\right.$ coil \#1) - $B$ (coil \#4), $\Delta B_{2}=B($ coil $\# 2)-B$ (coil \#3), and then $\Delta B=\frac{\left(\Delta B_{1}+\Delta B_{2}\right)}{2}$. In the above discussion, the contribution from the second term to $j_{p}$ is neglected because the equilibrium radial magnetic field is very small.

To obtain fluctuating $\mu_{0} \tilde{j}_{p}$ we also need to measure the second term of (3.2) because $\widetilde{B}_{r}$ is comparable to the other two components. On the "forked" probe, we have two $B_{r}$ coils toroidally separated, giving the value of the second term for $\tilde{j}$.

The method of $j$ measurement using the "forked" is a close analog to the method of measuring $j$ using the Rogowskii coil set. The coil array on the "forked" probe simulates a discrete portion of the Rogowskii coil set.

The size of each sensing coil is $2.5 \mathrm{~mm}$ in diameter, and the distance between two adjacent coils of the same oriertation is $1.1 \mathrm{~cm}$. This gives $\Delta x \geq$ 
$1.1 \mathrm{~cm}$, where $\Delta x$ is the differential distance when taking the gradient. Since the coil size is much smaller than $\Delta x$, the spatial resolution of the $j$ measurement can be optimized to the sub-centimeter range.

The absolute calibration of magnetic coils of the "forked" probe is done utilizing the Helmholtz coil set. By inserting the "forked" probe into the cavity of the Helmholtz set and applying a sinusoidal wave signal to the Helmholtz coil, the pick-up coils of the probe measure a voltage:

$$
\begin{aligned}
V_{\text {out }} & =-\frac{\partial \Phi}{\partial t}=-A_{\text {eff }} \frac{\partial B}{\partial t}=-A_{\text {eff }} \frac{\partial}{\partial t}[B \sin (\omega t+\alpha)] \\
& =-A_{\text {eff }}[\omega B \cos (\omega t+\alpha)]
\end{aligned}
$$

so that

$$
\left|V_{\text {out }}\right|=A_{\text {eff }} \omega|B|=k A_{\text {eff }} \omega\left|V_{\text {in }}\right|
$$

where $A_{\text {eff }}$ is the effective area of the pick-up coil that needs to be calibrated, and $k$ is some known constant coefficient relating $V_{i n}$. with $B$ for the Helmholtz set. 
As the frequency $\omega$ of the applied sinusoidal voltage varies, the ratio $\frac{V_{\text {out }}}{V_{\text {in }}}$ varies linearly with $\omega$ according to (3.4). From this linear relation, we get a very accurate result for $A_{e f f}$ Fig. 3.5 shows a typical example of such a linear relation. The slope of this curve is proportional to the effective area of the coil. The absolutely calibrated effective areas of the "forked" probe coils are about $1.60-1.62 \mathrm{~cm}^{2}$. This is very close to the physical area of the coil $\left(\pi r^{2} \mathrm{x}\right.$ No. of turns). The systematic error in calibration is small ( $\sim 1 \%)$.

The calibration linear relation between $\frac{V_{\text {out }}}{V_{\text {in }}}$ and $\omega$ does not hold for all frequencies. At very high frequency, the measured $\frac{V_{\text {out }}}{V_{\text {in }}}$ is smaller than the

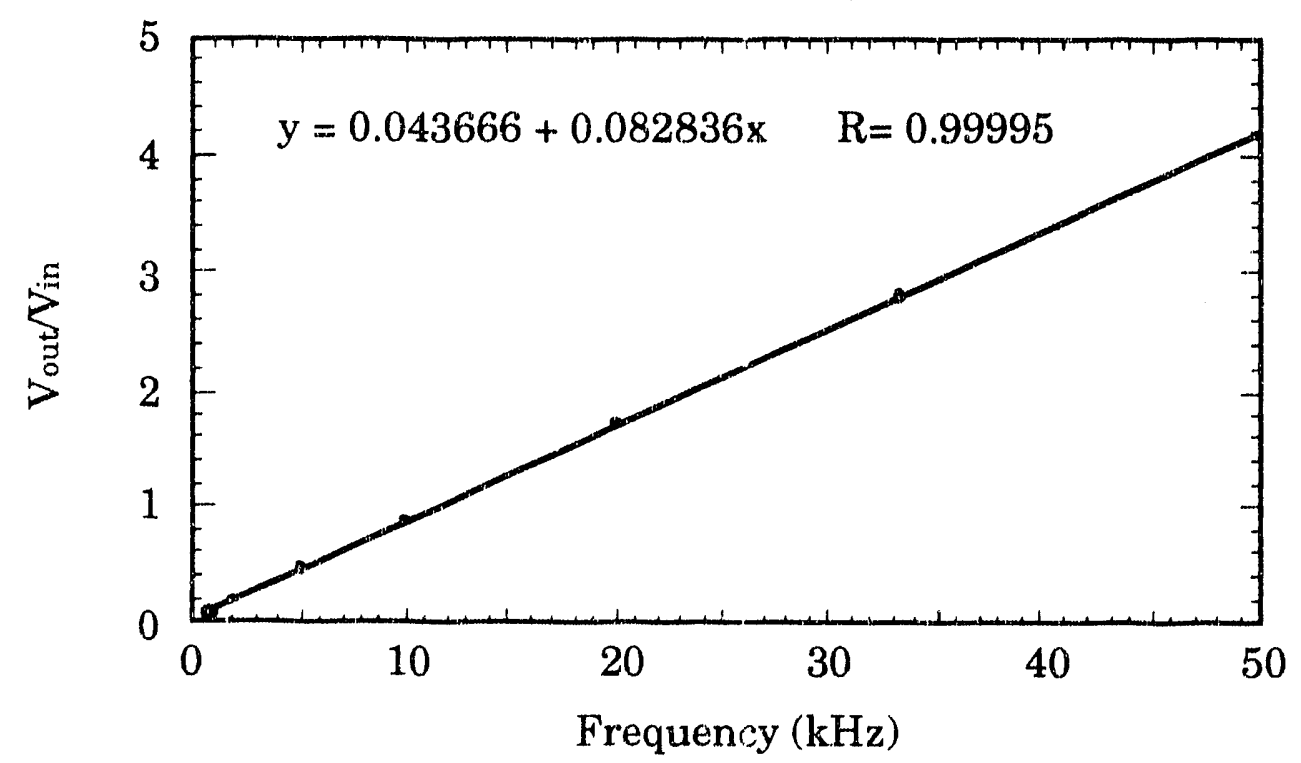

Fig 3.5 A typical plot of the calibration fit-curve for magnetic pick-up coils on the forked" probe. 
value expected by the linear relation. This is because the conducting (Stainless-Steel) shields restricts the penetration of high frequency electromagnetic fields, limiting the fields within a skin depth layer of the conductors. This causes a reduction of signal amplitude and a large frequency shift of the signals.

Upon insertion into the vacuum chamber, the probe has to be aligned relative to the coordinates of the machine. This is done by firing vacuum shots with only the toroidal field. The shots produce nearly uniform toroidal magnetic field across the minor radius of the chamber. An alignment with high precision can be accomplished by fixing the coils at such an angle that the $B_{t}$ pick-up coils detect maximum vacuum fieldsignals while the $B_{p}$ coils detect minimum signals. We estimate the experimental uncertainty in angular alignment is $0.5 \%-1 \%$. The vacuum toroidal field shots can also be used to double check the relative calibration of $A_{\text {eff }}$ by measuring the ratio of signals among all the coils.

Due to the small signal level from the magnetic pickup coils, the output signals are amplified. The electronic frequency responses of these signal amplifiers will affect the frequency characteristics of the measured signals. The stainless steel shields on the probe also affect the frequency response as 
stated above. The designed frequency response of the electronics is to flat between $1 \mathrm{kHz}$ and $300 \mathrm{kHz}$. The high frequency cutoff caused by the shield is around $200 \mathrm{kHz}$.

\subsubsection{Other Diagnostics for Turbulence and T'ransport Studies}

There are many other diagnostics incorporated in the experiment for measuring magnetic fluctuations and transport flux induced by these fluctuations, as well as the electrostatic fluctuation induced transport flux. There are a large number of magnetic pick-up coils residing on the inner wall of the conducting shell of MST forming arrays in both the poloidal and toroidal directions. These coil sets provide a good reference data base for the work of this thesis, and they are described in detail in reference ${ }^{4}$. The results of measuring electrostatic fluctuation induced transport have been presented elsewhere. ${ }^{5}$ 


\section{REFERENCES:}

1. R. N. Dexter et. al., Fusion Technology, 19, (1991), 131.

2 A. Almagri, Ph.D. thesis, University of Wisconsin-Madison.

$3 \quad$ D. C. Robinson, Nuclear Fusion, 18, (1978), 939.

4 S. Assadi et. al., Physical Review Letters, 69, (1992), 281.

$5 \quad$ T. D. Rempel et. al., Physical Review Letters, 67, (1991), 1438 
Chapter 4: Measurement of Plasma Current Density and Current Density Fluctuations

This chapter presents the results of measuring plasma current density and current density fluctuations in MST using the "forked" probe described in Chapter 3. Section 4.1 describes the measurement of equilibrium current density at the edge of MST. The application of equilibrium $j$ measurement in other experiments is presented. We will show that the "forked" probe indeed measures plasma current density, and it can be used in many important experiments involving current density modification. Section 4.2 describes the measurement of current density fluctuations for edge RFP plasmas. The result agrees with the global tearing mode simulation at low frequencies.

\subsection{Equilibrium Current Density Measurement in MST}

\subsubsection{Parallel Current Density Measurement in MST}

Since the current density in the parallel direction is the most important one for the study of confinement and transport, much of the work in this thesis is focused on the measurement of $j_{\| /}$. Furthermore, the parallel 
direction is very close to the poloidal direction for the edge of RFP plasmas. This is because the mean toroidal field goes to zero at the reversal surface while the poloidal field remains at some large value. The $j_{\| /}$and $\tilde{j}_{\|}$ measurements are approximated by $j_{p}$ and $\tilde{j}_{p}$ measurement.

Figure 4.1 shows the time evolution of the equilibrium parallel (poloidal) current density measured by the "forked" probe. The signal at each radial position is averaged over five identical shots to reduce the "sawtooth" oscillations. The equilibrium toroidal current density is also measured, and its amplitude is smaller by at least a factor of 4 . All the data were taken for discharges with $I_{p}=220 \mathrm{kA}$ and $F=-0.15 . F$ here is the field reversal parameter defined as: $F=\frac{B_{t}(a)}{\left\langle B_{t}\right\rangle}$.

Recently, there has been some work on the current density measurement accomplished on MST by using a movable Rogowskii probe. ${ }^{1}$ The probe has a small Rogowskii coil ( $2 \mathrm{~cm}$ in diameter ) mounted at the tip of the probe. The general characteristics of the measured equilibrium current densities by the two methods are very similar.

The plasma current density is one of the critical quantities in studying plasma confinement. With the help of reliable current density diagnostics such as the "forked" probe, many of the important experiments involving 


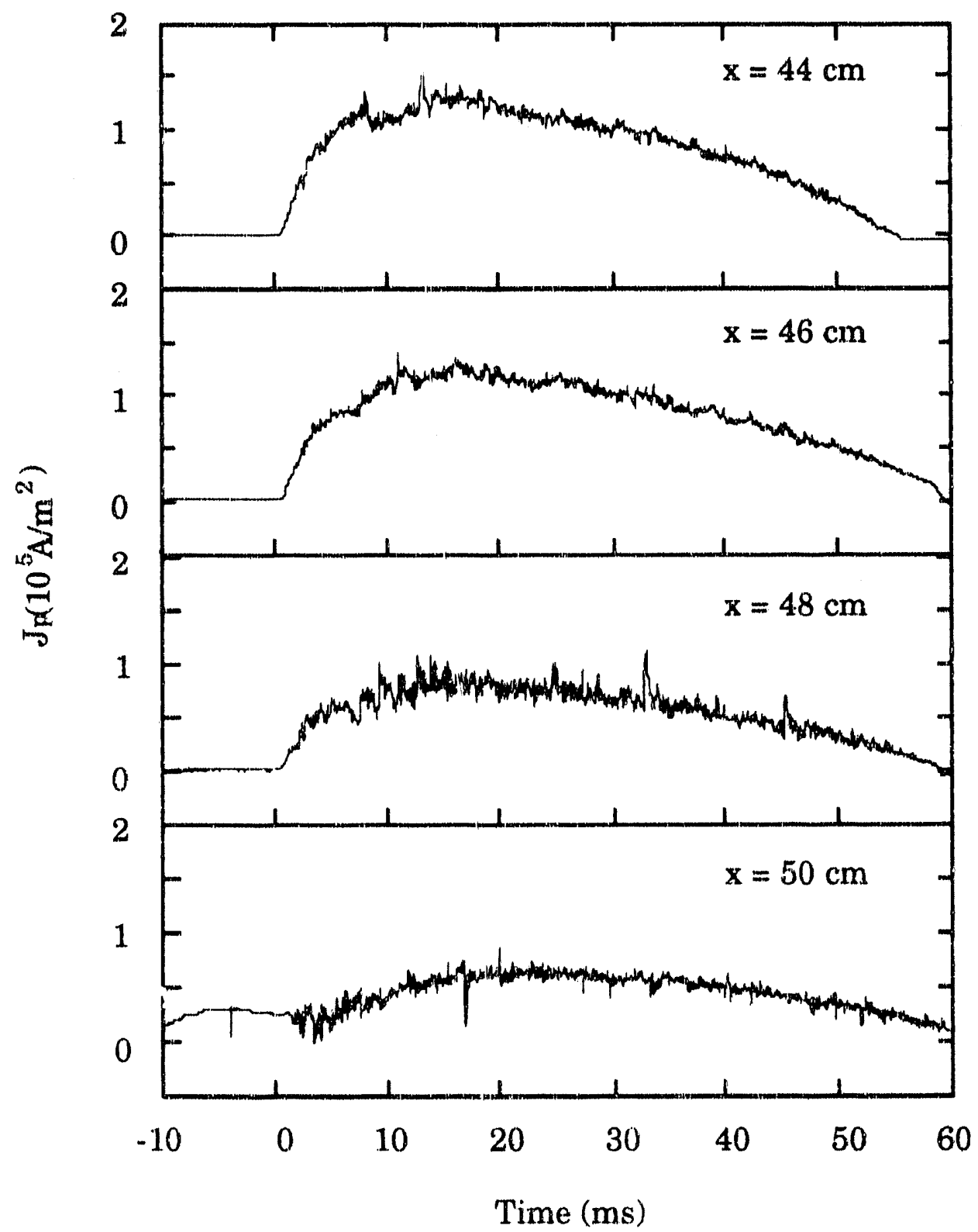

Fig. 4.1 The plasma current densities at the MST edge measured by the "forked" probe. Each of the traces is averaged over 5 identical shots. 
modification of the current density profile, current density injection, etc., could be performed with much better control. One such experiment is the pulsed-poloidal-ohmic-heating experiment.

\subsubsection{Current Density Measurement During PPOH Experiment}

The purpose of the pulsed-poloidal-ohmic-heating ( $\mathrm{PPOH})$ experiment is to flatten the current density profile at the edge of the RFP plasma. This will hopefully stabilize the global tearing instabilities. In normal RFP discharges, the profile of $\lambda_{/ /}\left(\lambda_{/ /}=\frac{\boldsymbol{j} \cdot \boldsymbol{B}}{B^{2}}\right)$ has a large gradient near the edge of the RFP plasma. This is partly due to the strong shear of the $B$ profile and partly due to the small $j_{/ /}$resulting from high resistivity near the edge. If, by some experimental means, one is able to increase locally the edge $j_{/ p}$ then $\mid \nabla$ $\lambda_{1 /} \mid$ would be reduced. This could affect the stability and the growth rate of global tearing modes that obtain their free energy from $\frac{d \lambda_{l l}}{d r}$.

The actual experimental procedure of $\mathrm{PPOH}$ is to add a pulsed, high voltage across the toroidal gap after the RFP plasmas are well established. This voltage is applied all the way around the long circumference of the torus to maintain the toroidal symmetry. This pulsed voltage generates a large poloidal current flowing at the inner wall of the conducting shell. This 
current in turn induces a corresponding poloidal current sheet in the edge of the plasma column. The direction of the induced shell current is in the direction to increase the field reversal at the plasma edge.

Fig. 4.2 and Fig. 4.3 are illustrations of the experimental setup and the circuit used in this experiment. The conditions of the plasma discharges used for the PPOH experiments are: $I_{p}=220 \mathrm{kA}, n \sim 1 \times 10^{19} \mathrm{~m}^{-3}$. For hardware reasons, this experiment can only be performed with the toroidal magnetic field kept at zero right at the wall. This is done by toggling the switch S2 in Fig. 4.3 off when starting the discharge. So these are $F^{\prime}=0$ discharges without the pulsed poloidal current. At $20 \mathrm{~ms}$ into the discharge, the power supply for the current pulse is turned on, giving a high voltage pulse at the toroidal gap of the torus.

Fig. 4.4 shows the time evolution of several important plasma quantities. They are (from top to bottom): toroidal gap voltage $\left(V_{t g}\right)$, average toroidal flux $\left(<B_{t}>\right)$, toroidal magnetic field at the wall $\left(B_{t}(a)\right)$ and poloidal plasma current density $\left(j_{p}\right)$. At $20 \mathrm{~ms}$, all quantities in Fig 4.4 show dramatic changes corresponding to the voltage pulse at the poloidal gap. The top trace is the toroidal gap voltage. A large negative voltage pulse is applied to the gap by the power supply at $20 \mathrm{~ms}$ for this experiment. This reduces the 


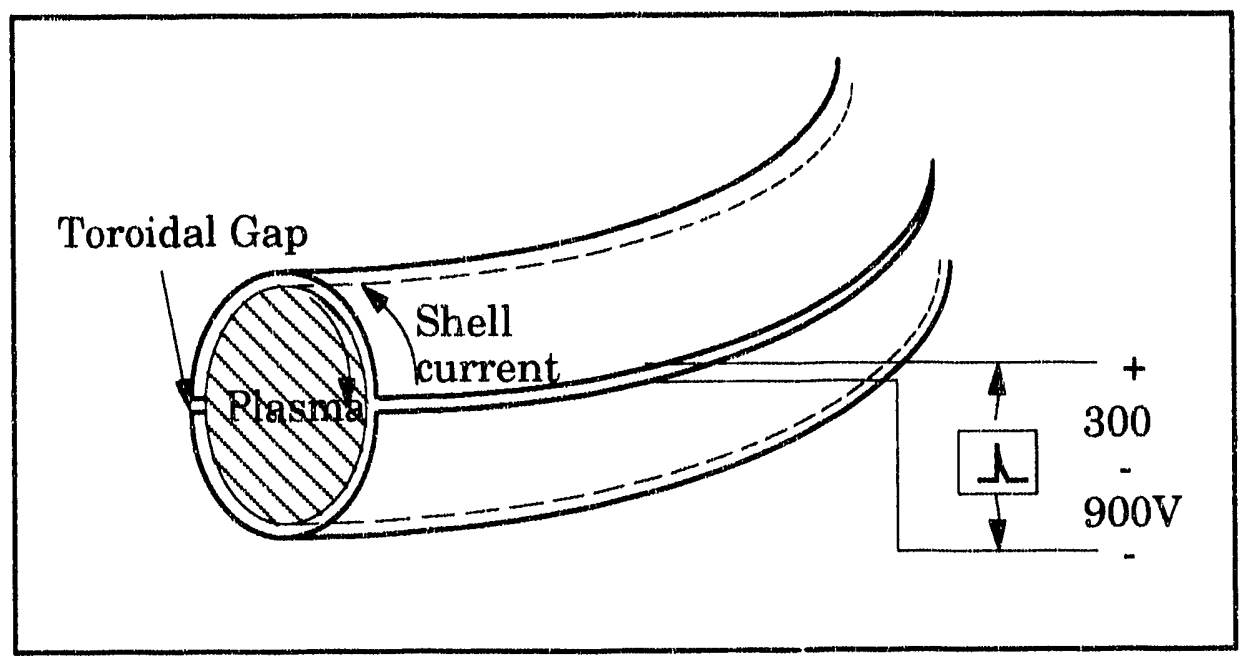

Fig 4.2 Schmatic illustration of the pulsed poloidal ohmic heating experiment.

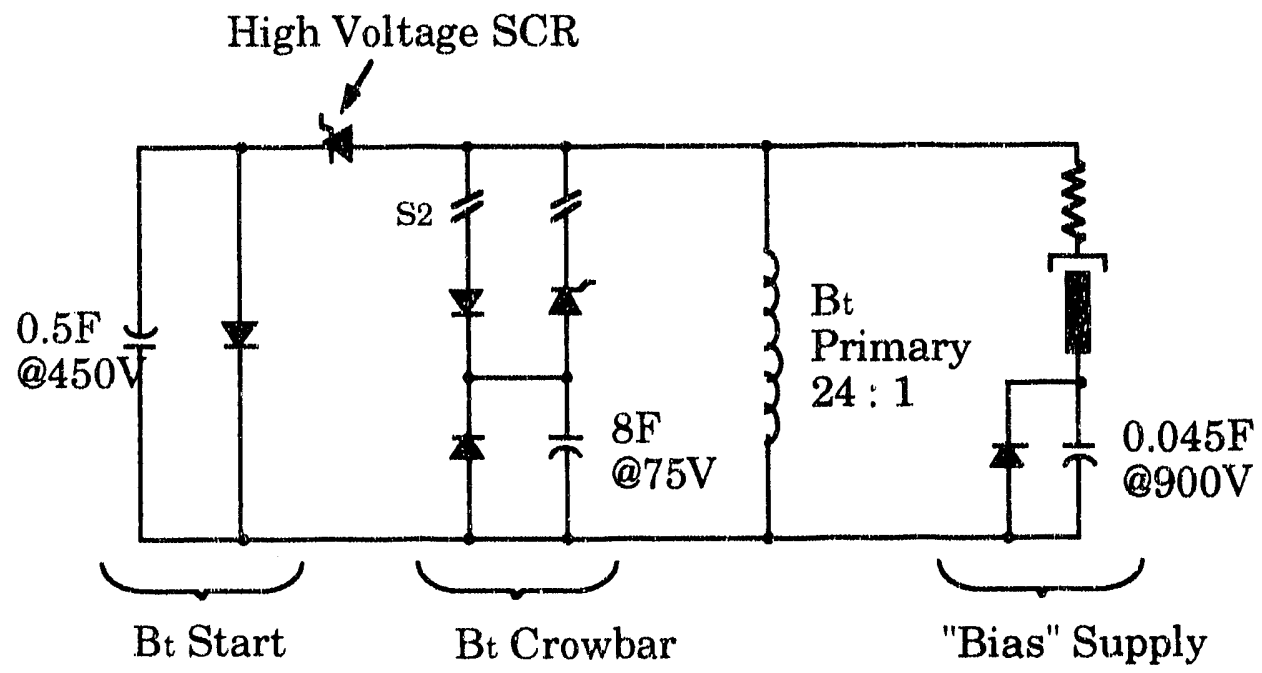

Fig. 4.3 The electric circuit used in the Vtg-pulser experiment. 


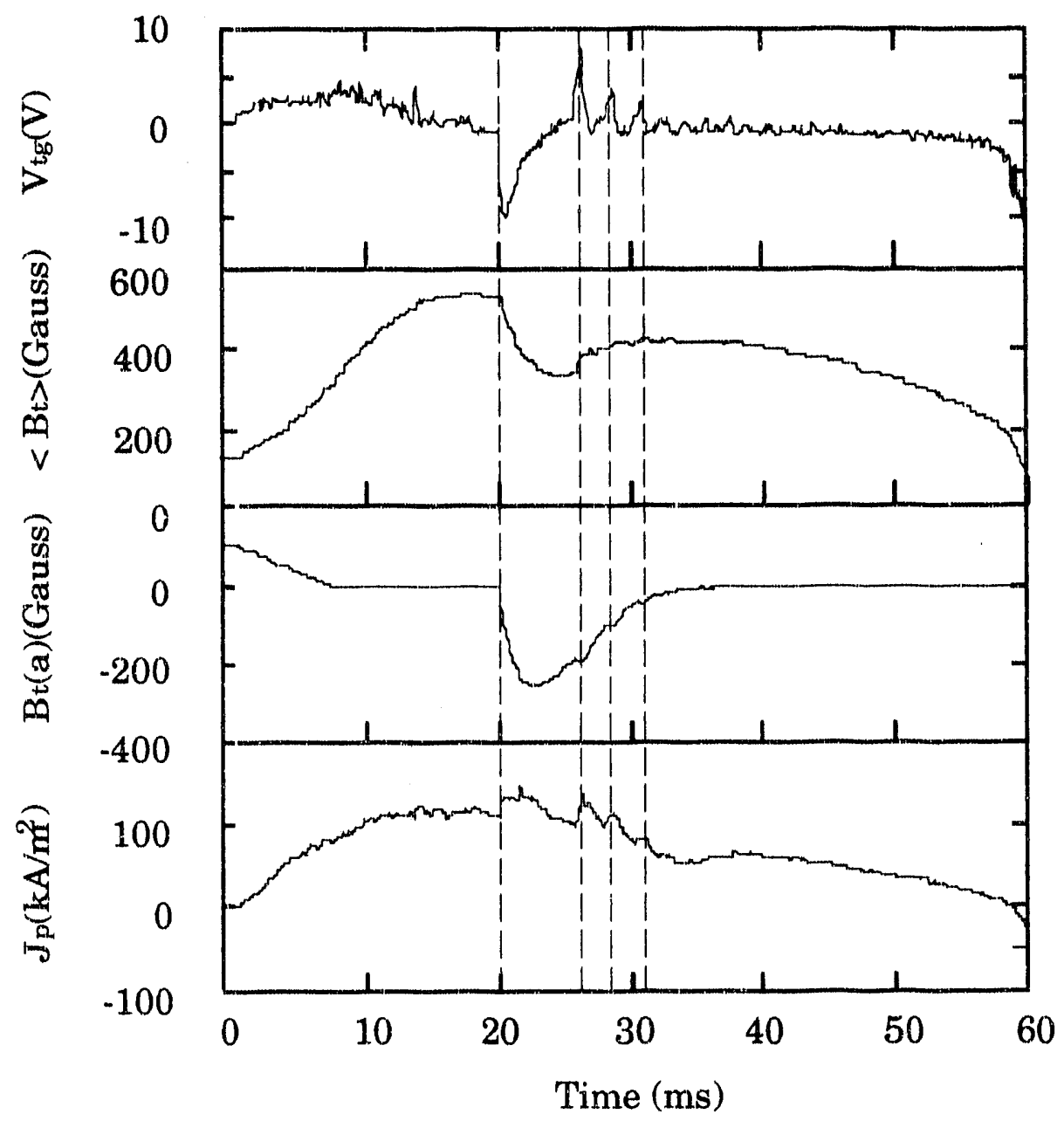

Fig. 4.4 A dramatic change corresponding to the pulse at Vtg on some of the important plasma quantities are clearly seen. The parameters plotted (from top to bottom) are: the toroidal gap voltage, the averaged toroidal magnetic flux, the toroidal magnetic field at the wall, and the poloidal plasma current density measured by the "forked" probe. 
overall toroidal flux as shown by the $\left\langle B_{t}>\right.$ trace. The reduction is due to the negative magnetic flux generated at the edge, shown by the $B_{t}(a)$ trace. The bottom trace is $j_{p}$ near the edge measured by the "forked" probe. It has a sudden increase as the pulsed current is applied.

Fig 4.5 is a typical time trace of the poloidal current density measured by the "forked" probe. The signals are averaged over five shots with nearly identical discharge conditions. We see that $j_{p}$ is very similar to that in Fig. 4.1 except for the sudden increase at $20 \mathrm{~ms}$ as the Vtg-pulser is fired.

To see the profile flattening effect of the pulsed poloidal current, the values of $j_{p}$ at several radial positions near the plasma edge are plotted in Fig. 4.6. Fig. 4.6 (a) and (b) are the partial profiles of $j_{p}$ before and after the pulsed current induced respectively.

Before the PPOH power is turned on, the parallel current density profile is peaked with decreasing $j_{/ /}$towards the edge. This is illustrated in Fig. 4.6 (a). This profile is maintained for many milli-seconds by the dynamic process associated with the "dynamo". As the power supply pulses, the $j_{p}$ profile has a "bump" appearing at the very edge. Thus the profile at this edge region is significantly flattened as shown in Fig. 4.6 (a). As time progresses, this "bump" propagates inward, as indicated in Fig. 4.6 (b). This inward 


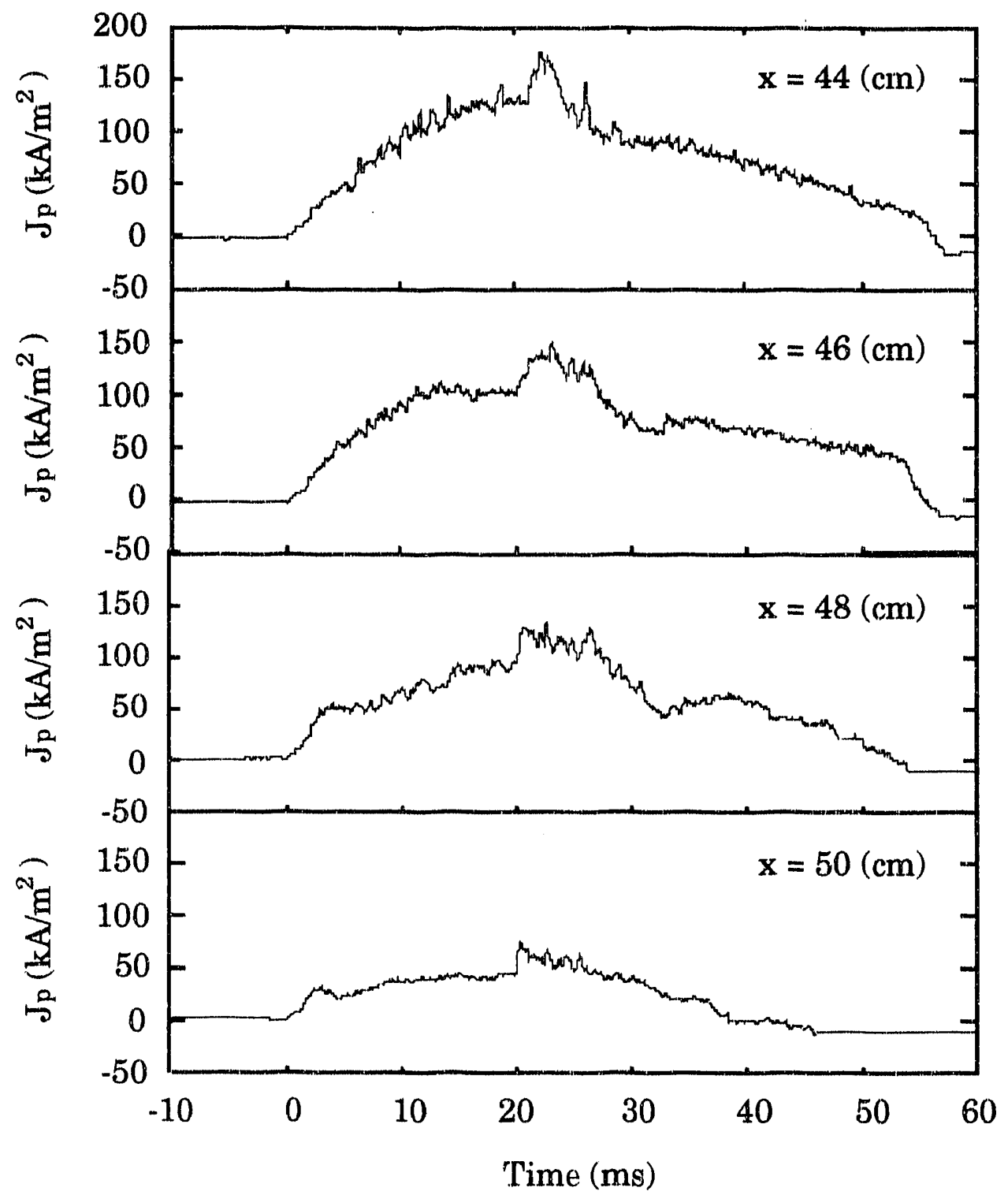

Fig. 4.5 Edge plasma current density at four radial locations. Note the sudden increase at $20 \mathrm{~ms}$ as the Vtg-pulser fires. 


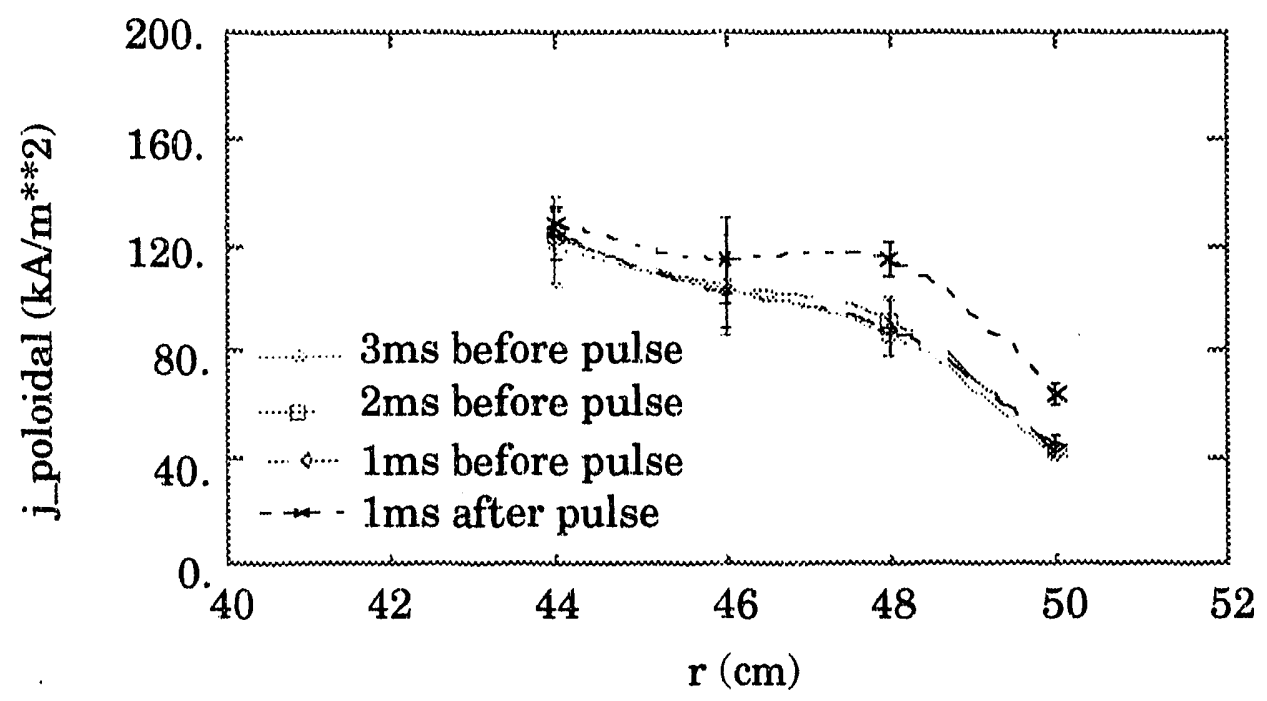

(a)

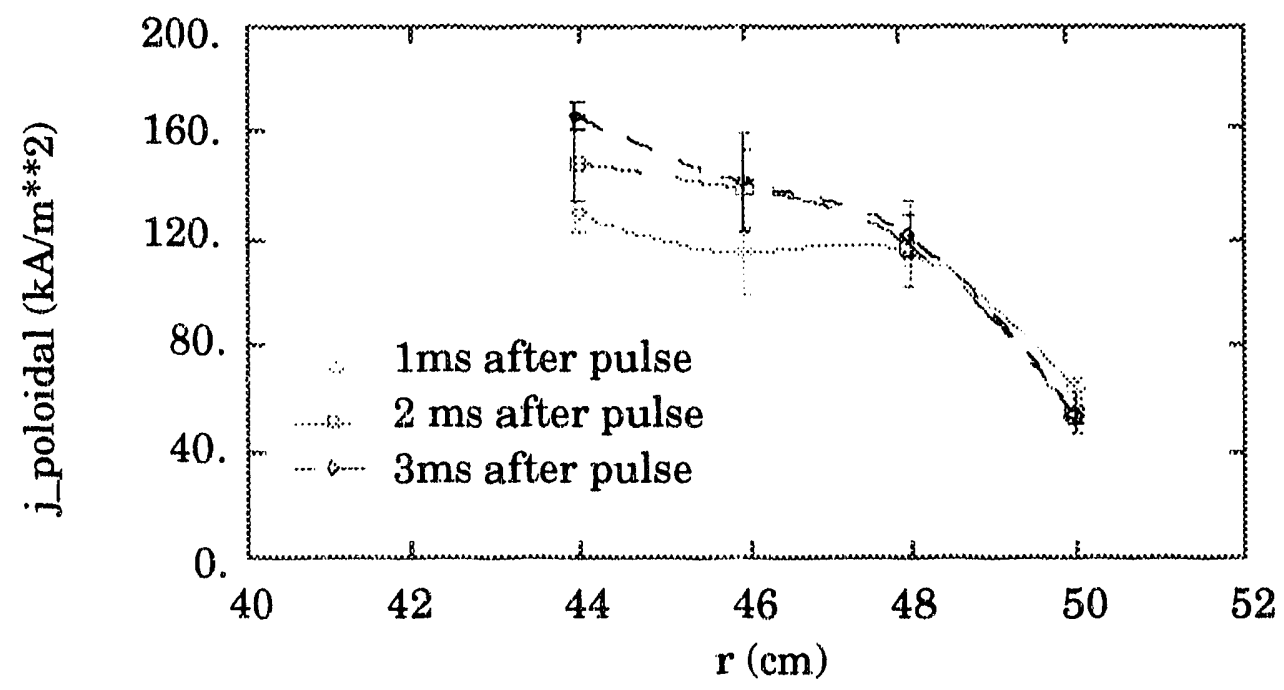

(b)

Fig. 4.6 The profile change (flattening) due to the pulse voltage applied at the toroidal gap of the torus. 
propagation of current density is probably caused by the diffusion process. The diffusion rate is $\sim 20 \mathrm{~m}-\mathrm{s}^{-1}$.

We have demonstrated through the $\mathrm{PPOH}$ experiment the importance of the "forked" probe as a relialile current density diagnostic tool. Further studies of current driving and current injection for the RFP plasmas in the future would largely depend on the current density diagnostics with high accuracy. The "forked" probe is one such diagnostic, subject to many improvements though.

\subsection{Current Density Fluctuation Measurement on the MST}

To study the anomalous transport of plasma particles induced by magnetic fluctuations, it is critically important to measure the plasma current density fluctuations. The expressions of particle fluxes obtained in equations (2.2) and (2.3) predict a direct dependence of these fluxes on the parallel current density fluctuations, and their coherence with radial magnetic field fluctuations. Current density fluctuation measurements also provide an additional tool for identifying the modes of magnetic fluctuations. In this section, we report the measurement of parallel current density 
fluctuations performed on MST. The transport study from measured $\tilde{j}$ and $\tilde{b}$ is presented in chapter 6 .

Figure 4.7 shows a time trace of the fluctuating poloidal current density measured by the "forked" probe. The data were taken for a typical RFP plasma shot with $I_{p}=220 \mathrm{kA}$. Also shown are three components of the magnetic fluctuations measured by the same probe. Coherent oscillatory structures with $f \sim 15 \mathrm{kHz}$ appear on the time traces of all $\widetilde{B}$ and $\tilde{j}$.

Fig 4.8 is the frequency power spectrum of $\tilde{j}_{p}$ at $r=46 \mathrm{~cm}$. 'The result is obtained by a statistical spectral analysis method discussed in the Appendix. The number of terms used in the ensemble average is 256 . These 256 data points correspond to $0.5 \mathrm{~ms}$ of signal. A strong peak with $f \sim 15 \mathrm{kHz}$ is seen corresponding to the coherent structure in Fig. 4.7. The $\tilde{j}_{p}$ power spectrum indicates that more than $90 \%$ of the power is from the fluctuations with $f<$ $50 \mathrm{kHz}$. This is consistent with the resistive tearing mode predictions. The spectrum becomes broader than the magnetic fluctuation spectra at high frequency, probably due to the non-linear dispersion relation between $\omega$ and $k$. This is because $j_{(\omega)} \sim k(\omega) B_{(\omega)}$ and $k_{(\omega)}$ is in general broader as $\omega$ increases. From the power spectrum, the aniplitude of $\tilde{j}_{p}$ is obtained at $\sim 15$ $\mathrm{kA} / \mathrm{m}^{2}$. 


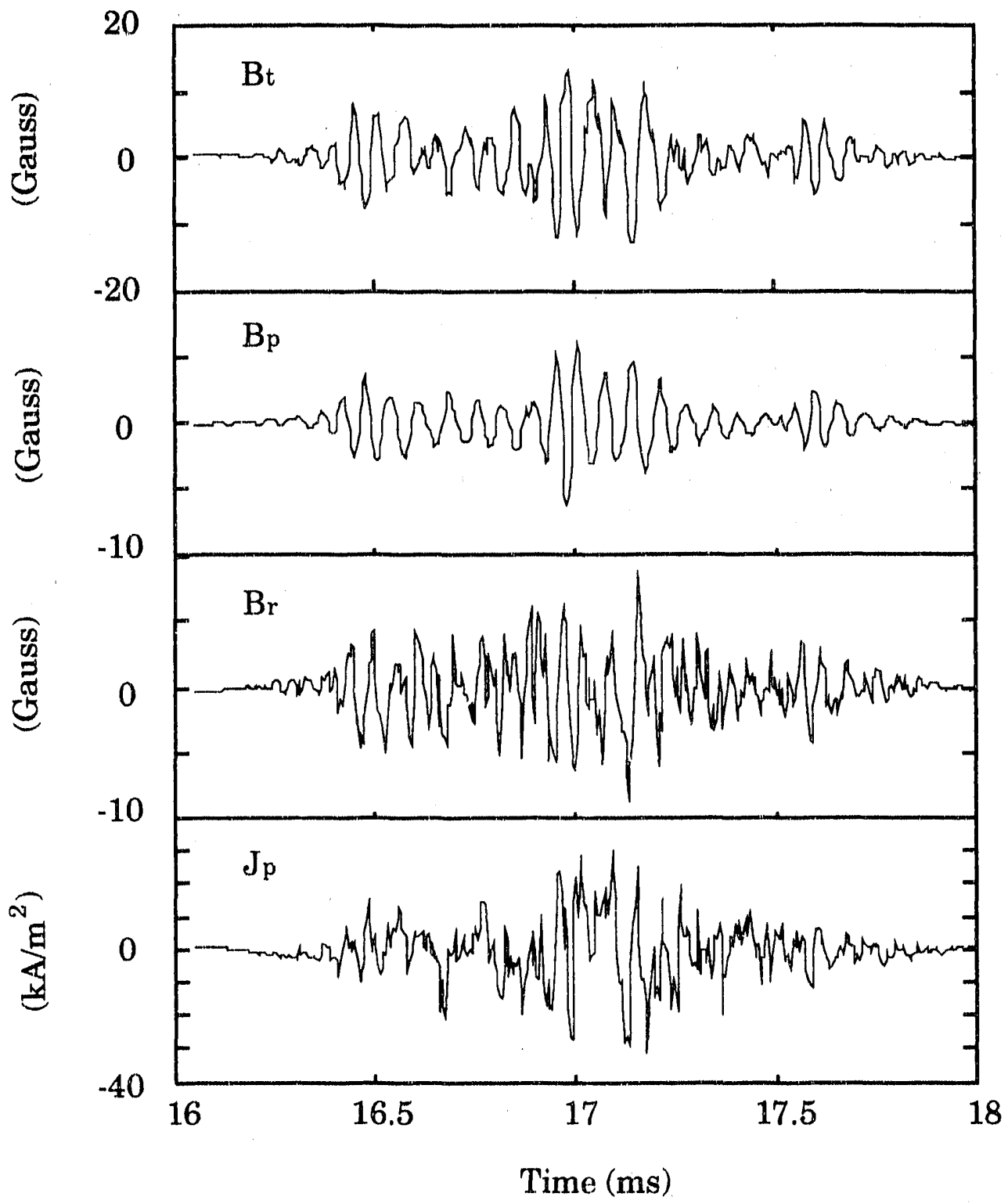

Fig. 4.7 Measured plasma current density fluctuations at $r=$ $46 \mathrm{~cm}$. Also plotted are 3 components of the magnetic field fluctuations. Low frequeny coherent oscillations can be seen on both $B$ and $j$. 


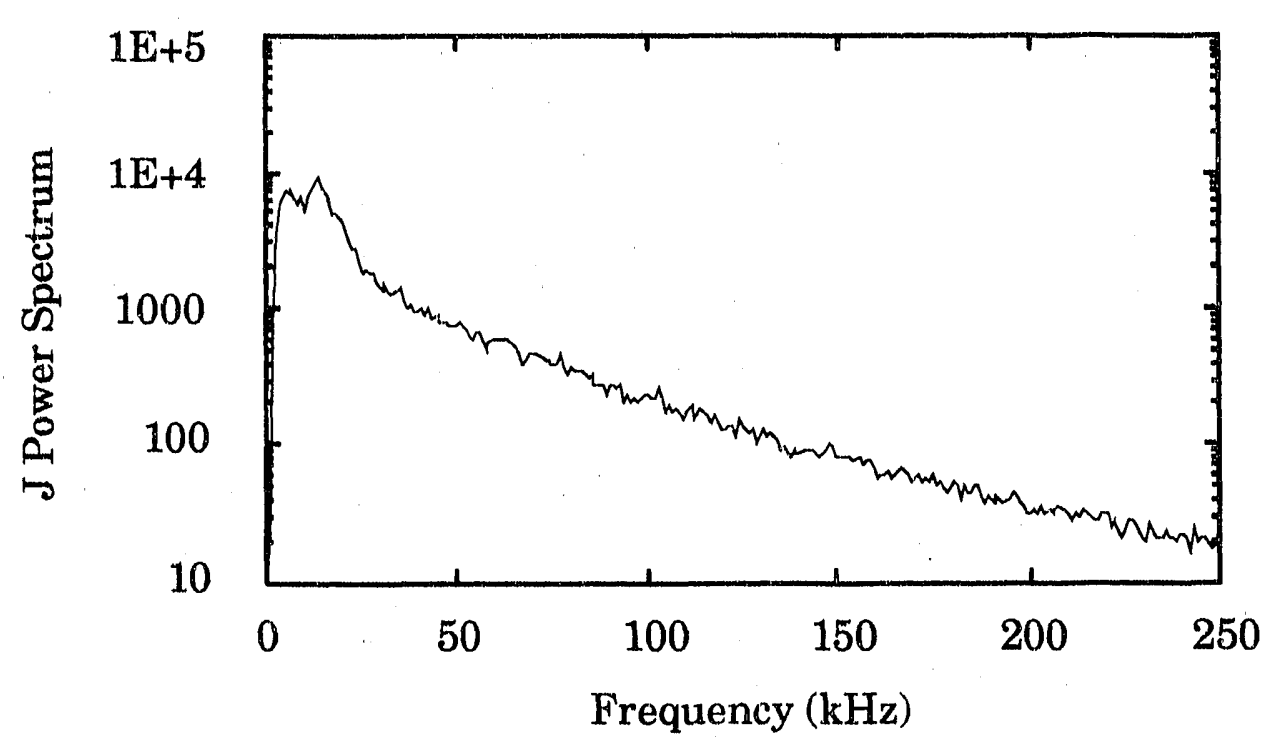

Fig. 4.8 Power spectrum of $J_{p}$ at $x=46(\mathrm{~cm})$.

The fractional fluctuation amplitude of plasma current density is calculated as $\frac{\tilde{j}_{\| \prime}}{j} \sim \frac{\tilde{j}_{p}}{j_{p}} \sim 8-10 \%$ from Fig. 4.1 and 4.8. The toroidal current density fluctuation is about one third to one half of the poloidal current density fluctuation. The measured $\tilde{j}_{p}$ amplitude is a strong function of plasma radius. The $\tilde{j}_{p}$ amplitude increases rapidly as the probe is moved inward.

The level of the current density fluctuation fraction $\frac{\tilde{j}}{j}(\sim 8-10 \%)$ is much higher than the magnetic fluctuation fraction $\frac{\widetilde{B}}{B}(\sim 0.5-1 \%)$. Also, both $\frac{\tilde{j}}{j}$ 
and $\frac{\widetilde{B}}{B}$ are higher than their predicted values from 3-D MHD code by the same factor $(\sim 3)$. This could be because the code uses an unrealistically low magnetic Reynolds number $S$. The unbearably long computing time and high cost for computing a high $S$ case limit the simulation results to $S$ less than $10^{4}$. The experimental $S$ value is $\sim 10^{6}$.

RFP plasmas are believed to be operated at close to a force-free low- $\beta$ state, that is: $\boldsymbol{j} \times \boldsymbol{B} \approx 0$. For equilibrium magnetic field and plasma current density, $\boldsymbol{j}$ parallel to $\boldsymbol{B}$ is a good approximation. On the other hand, the fluctuating magnetic field and current density are not parallel to each other, as the measurement demonstrates: $\frac{\widetilde{B}_{t}}{\tilde{B}_{p}} \approx 2$, and $\frac{\tilde{j}_{t}}{\tilde{j}_{p}} \approx 0.5$ for low frequency fluctuations.

The expression of $\tilde{j}_{p}$ according to Ampere's law is

$$
\mu_{0} \tilde{j}_{p}=\frac{\partial \tilde{B}_{t}}{\partial r}-\frac{1}{R}\left(\frac{\partial \tilde{B}_{r}}{\partial \phi}\right)
$$

At the RFP plasma edge, the second term in (4.1) has larger contribution to $\tilde{j}_{p}$ since the radial gradient of $\widetilde{B}_{t}$ is very small. It is estimated from the measurement that the $\left|\frac{1}{R}\left(\frac{\partial \widetilde{B}_{r}}{\partial \phi}\right)\right|$ term contributes $70-80 \%$ of the $\left|\tilde{j}_{p}\right|$.

Even though the experimental uncertainty of measuring $B$ is as small as 
$1 \%$ (see discussions in Chapter 3), the measument error bars can be very large for $j$ and $\tilde{j}$ measurements. This is because taking gradients by substracting two similar signals can amplify the small errors of individual signal by a large factor. We estimate the experimental uncertainties of $j_{p}$ and $\tilde{j}_{p}$ are $5 \%-7 \%$ and $12 \%-15 \%$, respectively, compared to less than $1 \%$ for $B$ and 3 


\section{REFERENCES}

1 A. F. Almagri, Private communication. 
Chapter 5: Measurement of Internal Magnetic Fluctuation and Mode Number Spectrum

This chapter presents the results of internal measurements of magnetic fluctuations in the RFP plasmas in MST. Section 1 contains the description of general features of internal magnetic fluctuations. Cornparison with the external measurements by a fixed pick-up coil array will be given. Section 2 describes the measurements of mode number spectra using the two-point correlation method. The dependence of such spectra on radial positions in the plasma is investigated for low and high frequency magnetic fluctuations, respectively.

\subsection{Internal Magnetic Fluctuations in MST}

The magnetic fluctuations have been measured internally using the "forked" probe with multiple magnetic sensing coils inserted in MST. Fluctuating magnetic signals of all three components with frequency up to $250 \mathrm{kHz}$ are neasured inside the RFP plasmas. This section describes some of the characteristics of internal magnetic fluctuations. Section 5.1.1 revisits 
the results of external measurements by the fixed coil array. These results have been presented by others before. ${ }^{1}$ Section 5.1 .2 gives new results of internal measurements of magnetic fluctuations. Comparison with the results in 5.1 .1 will be made.

\subsubsection{General Characteristics of Externally Measured Magnetic Fluctuations}

Before we present the results of internal measurements of magnetic fluctuations on MST, it is useful to review some of the results from the external magnetic fluctuation measuremen s made by the fixed coil array. Interested readers are encouraged to reac reference 1 for more detailed information.

The magnetic fluctuations with frequency from $1 \mathrm{kHz}$ to $250 \mathrm{kHz}$ have been measured externally by a large number of fixed pickup coils, forming arrays in both poloidal and toroidal directions, at the inner wall of the

conducting shell of MST. The coil sets are evenly distributed up to 64 coil sets toroidally, and 16 coil sets poloidally, with three coils facing three directions on each coil set.

The measured frequency spectra of magnetic fluctuations are dominated by the low frequency portion. More than $90 \%$ of the power is from 
fluctuations with frequencies below $50 \mathrm{kHz}$. The amplitude relation among three components is: $\widetilde{B}_{t}: \widetilde{B}_{p}: \widetilde{B}_{r}=1: 0.5: 0.2$ for those low frequency fluctuations.

Statistical spectral analysis has shown that the dominant mode spectra are: $m=1, n=6,7,8$, and $\Delta n<n$. The positive value of $\frac{m}{n}$ here corresponds to internally resonant modes. These dominant modes have the characteristics of resistive tearing modes predicted by many theoretical and numerical models for RFPs, as the models predict $m=1, n \sim \frac{2 R}{a}$. For MST, the aspect ratio $\frac{R}{a}$ is close to 3 . A helical rotation of $m=1$ fluctuations with rotating velocity close to the electron diamagnetic drift velocity is detected.

At high frequency ( $f$ between $50 \mathrm{kHz}$ and $250 \mathrm{kHz}$ ), the magnetic fluctuations show significantly different characteristics compared with the low frequency case. The amplitudes have been reduced by two orders of magnitudes. Also, the measurement of mode number spectra indicates that the peak of the $n$ spectrum shifts to the negative side, corresponding to external resonant modes.

\subsubsection{General Features of Irternal Magnetic Fluctuations in MST}


Magnetic fluctuations of all three components have been measured at the plasma edge $\left(\frac{r}{a}>0.85\right)$ inside MST using the "forked" probe. Figure 5.1 shows the $\dot{B}$ signals for toroidal, radial and poloidal components at $r=46 \mathrm{~cm}$ for a typical dischargo with $I_{p}=220 \mathrm{kA}$. At the bottom of Fig. 5.1 are the time traces of the equilibrium toroidal field (averaged over the entire torus ) at the conducting wall $B_{t}(a)$ and the average toroidal magnetic field $<B_{t}>$. The low frequency coherent oscillations are clearly present during $13.6-14.3$ ms on $\dot{B}$ signals for the shot presented. Superimposed on these coherent oscillations are high frequency fluctuations with smaller amplitudes. Also present are sudden "bursts" of signals as the plasma experiences "sawteeth" activity that is signified by a sudden increase in $\left\langle B_{t}\right\rangle$.

Fig. 5.2 are the power spectra of magnetic fluctuations of the three components at $r=46 \mathrm{~cm}$. A clear peak is seen for $15 \mathrm{kHz}<f<30 \mathrm{kHz}$, corresponding to the coherent structures seen in Fig. 5.1. On the other hand, the high frequency portion of the spectra indicates a broad-band turbulence with no dominant peaks present. We will treat these two parts of the spectra separately since they are significantly different in many aspects.

The data used to calculate the fluctuation power spectra in Fig. 5.2 are taken near the peak of the plasma current. 


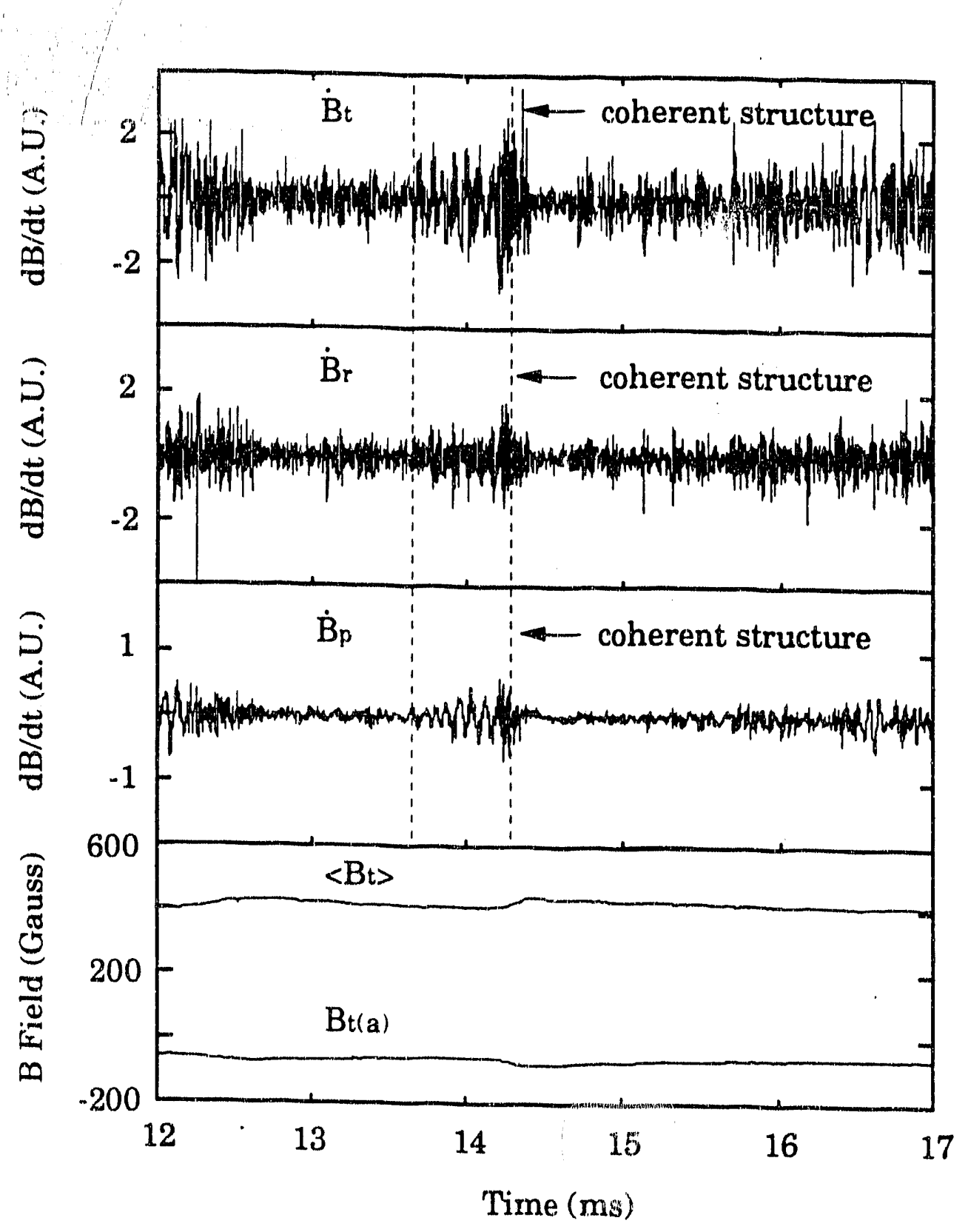

Figure 5.1 Raw signals from magnetic pickup coils of "the Forked" probe. Also shown are the equilibruium toroidal magnetic field at the wall and the average toroidal magnetic field. The coherent oscillations can be seen clearly, particularly on the $d B p / d t$ signal. Note the jumps on the equilibrium signals. 


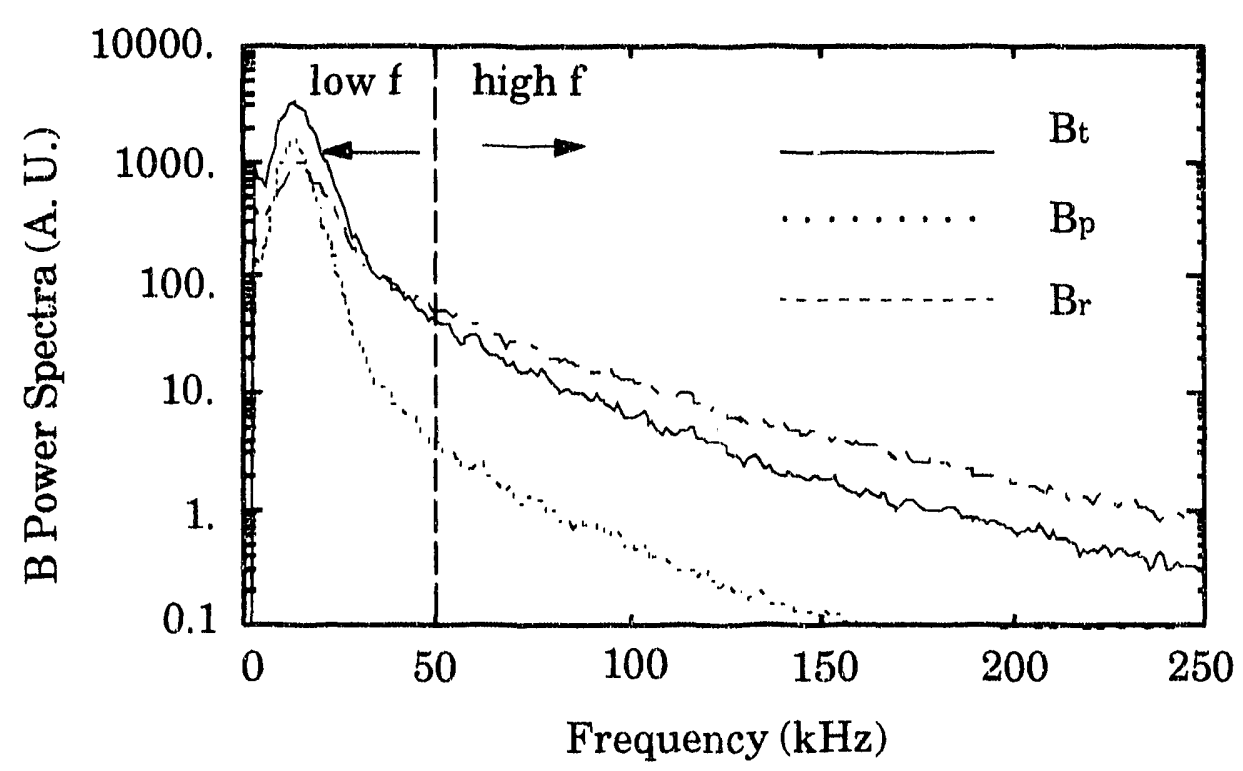

Figure 5.2 The power spectrum of magnetic fluctuations for all 3 componentes are strongly peaked at $f \sim 15 \cdot 30(\mathrm{kHz})$.

The fluctuation amplitude relation among three components is obtained as: $\widetilde{B}_{t} \sim 2 \widetilde{B}_{p} \sim 5 \widetilde{B}_{r}$ for low frequency magnetic fluctuations, as demonstrated in Fig. 5.2. Measurements done at other radial locations inside the plasma have given similar results. This amplitude relation is in agreement with results from external measurements (See ref. 1 or section 5.1.1 of this thesis). Fig. 5.2 also indicates that more than $90 \%$ of magnetic fluctuation power is concentrated at frequencies below $50(\mathrm{kHz})$.

The amplitude relation among the three components at high frequency 
$(f>50 \mathrm{kHz})$ is very different from the low frequency case. The parallel component of the magnetic fluctuation level decreases much faster than the other two components as the frequency increases. This can be clearly seen in Fig. 5.2 by noting that the parallel direction is very close to the poloidal direction for the edge of the RFP plasma. The radial magnetic fluctuation becomes the dominant one at high frequency. Quantitatively, we have the amplitude relation for high frequency fluctuations: $\widetilde{B}_{r} \sim 2 \widetilde{B}_{t} \sim 10 \widetilde{B}_{p}$. This character is in fact very similar to the magnetic fluctuations in tokamaks where $\tilde{B}_{r} \gg \tilde{B}_{\| /}$, and $\frac{\widetilde{B}}{\bar{B}} \sim 10^{-4}-10^{-5}$. It should be mentioned that this amplitude relation is different from the external measurement where $\widetilde{B}_{r}$ is the smallest.

Figure 5.3 shows the spectra of cross-coherence $\gamma$ and cross-phase $\Delta \phi$ between $\widetilde{B}_{t}$ and $\widetilde{B}_{r}, \widetilde{B}_{t}$ and $\widetilde{B}_{p}$. Also plotted are $\gamma$ and $\Delta \phi$ between two $\widetilde{B}_{t}$ signals separated by $1 \mathrm{~cm}$. The definition of $\gamma$ and $\Delta \phi$ is described in the Appendix.

The peak values of the cross-coherence between $\widetilde{B}_{t}$ and $\widetilde{B}_{r}$, and between $\widetilde{B}_{t}$ and $\widetilde{B}_{p}$ at low frequency are well above 0.9 . This is the natural result of low frequency, global magnetic fluctuations. Similar results are reported from many other RFP devices (See Chapter 2). The general features of 


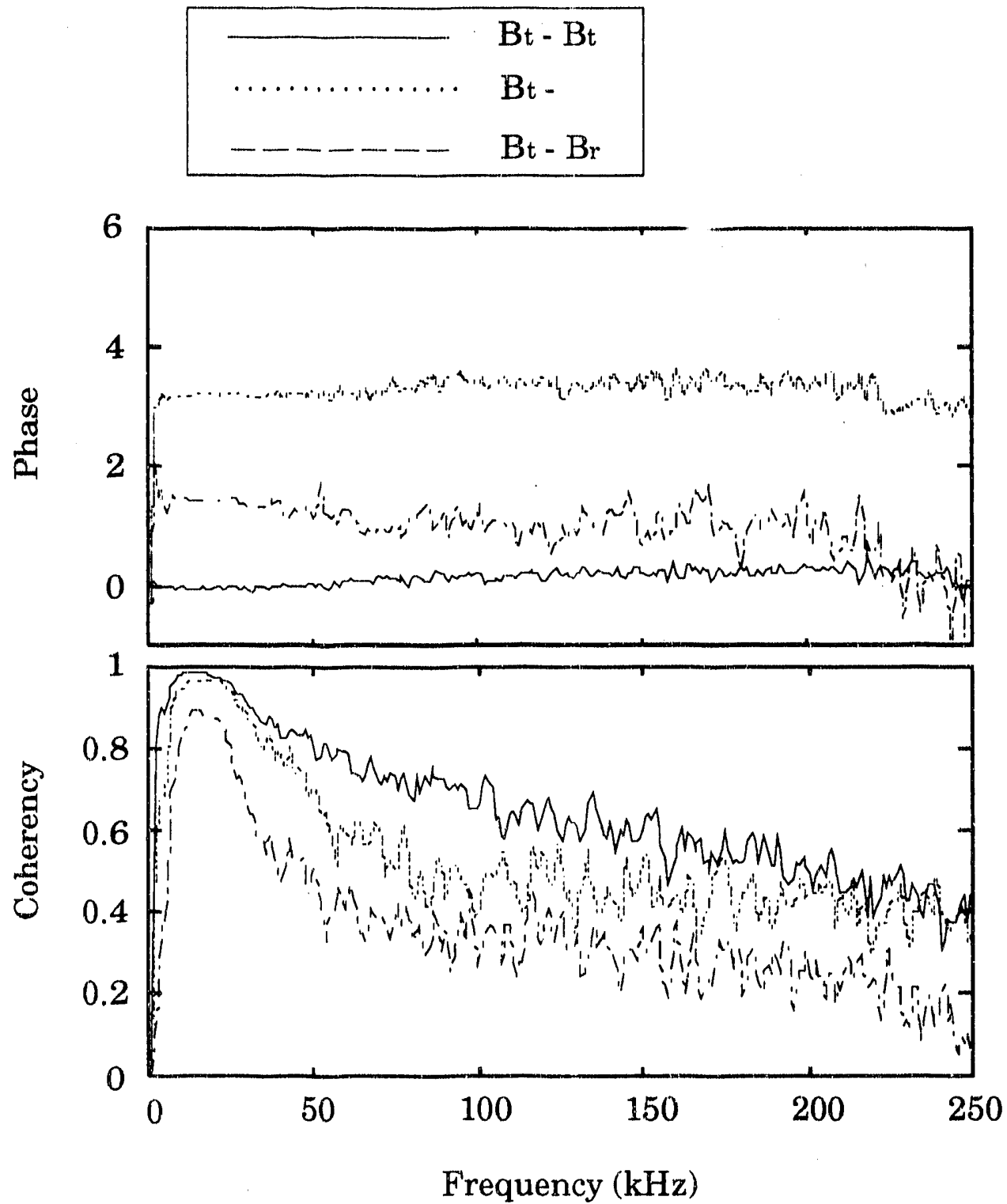

Fig. 5.3 The results from statistical spectral analysis schemes show the exsistence of high cross-coherence and clearly defined cross-phase angle among 3 components of fluctuating $B$. 
these global magnetic fluctuations satisfy the description of the resistive tearing instability discussed in Chapter 2. In Chapter 6 we will show that the mode number spectra for internal magnetic fluctuations agree with the predictions of the tearing mode model as well.

The result in Figure 5.3 has no dependence on the radial positions, at least within the outer portion of the plasma column $\left(0.85<\frac{r}{a}<1\right)$. The value of $\gamma$ decreases with an increase of frequency. At $f=250 \mathrm{kHz}$, we have $\gamma$ $\geq 0.15$.

Even though the spectra of cross-coherence $\gamma$ between each pair of the three components have similar frequency characteristics, their aross-phases, $\Delta \phi$, take different values, as seen in Fig. 5.3. At low frequency, the relation is: $\Delta \phi_{t, r}=\frac{\pi}{2}, \Delta \phi_{t, p}=\pi$. This cross-phase relation is as predicted for resistive tearing modes. ${ }^{2}$ The experimentally measured phase relation for dominant fluctuations agrees with the phase relations of the tearing instabilities predicted by a 3-D MHD code for RFPs.

The low frequency magnetic fluctuations are measured to have helical propagations, as shown by a constant cross-phase for frequency between 10 $\mathrm{kHz}$ and $35 \mathrm{kHz}$. The phase velocity of such propagation is defined as: $v_{\phi}=$ $\frac{2}{k} \frac{\pi f}{k}=2 \pi f\left(\frac{\Delta x}{\Delta \phi}\right)$. Although $v_{\phi}$ varies from shot to shot, it falls in the range 
close to the electron diamagnetic speed. Outside the reversal surface, the propagation phase velocity is in the ion diamagnetic drift direction. This is in contrast with the result from the OHTE RFP device. ${ }^{3}$ In OHTE the $m=1$ modes are detected to propagate in the electron diamagnetic drift direction.

No radial propagation of dominant modes is detected since $\Delta \phi_{t 1, t 2}=0$. The low frequency magnetic fluctuations of this type are said to have the structure of a radial standing wave. The phase of oscillations is the same over large radial scale lengths.

\subsection{Global and localized magnetic fluctuations in MST}

In this section we will give detailed results of the mode number spectrum measurements. We will show the difference in the radial dependence of the mode number spectra for low and high frequency fluctuations. This radial dependence gives further evidence of global and localized modes at different frequencies.

First, to determine the spatial, structure of the measured magnetic fluctuations, the radial coherence length $L_{r}(\omega)$, defined as $L_{r}(\omega)=-\frac{\Delta x}{\ln \left[\gamma_{\Delta x}(\omega)\right]}$, is calculated. This quantity is plotted as a function of frequency in Figure 


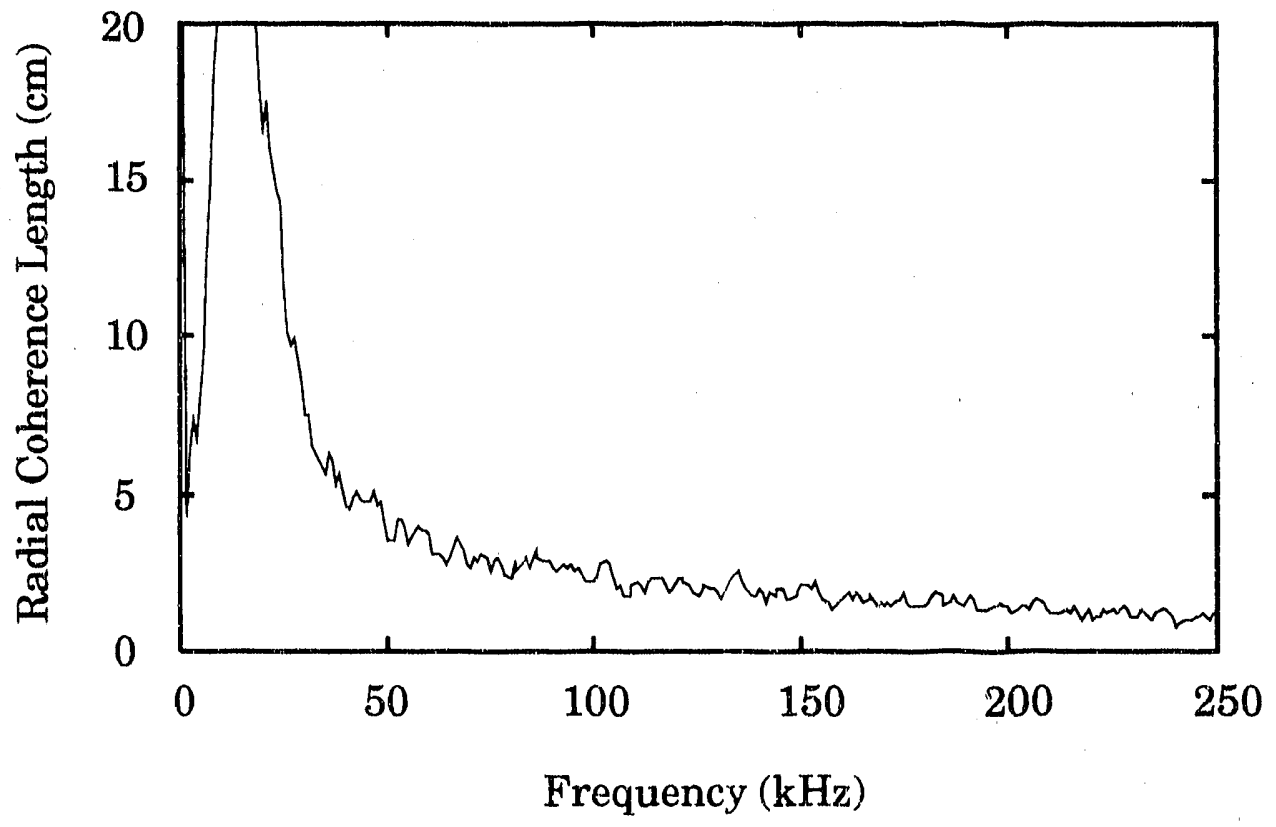

Fig. 5.4 Radial coherence length derived from two Bt pickup coils radially separated by $1 \mathrm{~cm}$.

5.4. Here $\gamma_{\Delta x}$ is the measured cross-coherence between two magnetic signals that are radially separated by $\Delta x$. We see in Fig. 5.4 that $L_{r}(\omega)$ is very sensitive to the fluctuation frequency. In the low frequency range $(f<$ $50 \mathrm{kHz}), L_{r}(\omega)$ has a value of $30-40 \mathrm{~cm}$. This is close to the plasma minor radius for the MST plasmas. As froquency increases to $50 \mathrm{kHz}$ and above, the value of $L_{r}(\omega)$ decreases very quickly. At $f>100 \mathrm{kHz}, L_{r}(\omega)$ has fallen by a factor of 10 or so, to a value of $1-3 \mathrm{~cm}$.

The further evidence of the global and localized modes in different 
frequency ranges for the magnetic fluctuations is obtained from the mode spectrum analysis. The method used to calculate the mode number spectrum is discussed in some detail in the Appendix. Basically, this method allows the estimation of the power weighted mode number $n(m)$ spectrum by analyzing the turbulent time signals taken from two spatial points that are toroidally (poloidally) separated.

Figure 5.5 and 5.6 are the $n$ and $m$ spectra at two different radial locations, $x=42 \mathrm{~cm}$ and $x=46 \mathrm{~cm}$. Previous magnetic probe measurements have determined that for the discharge conditions stated above, the position of the reversal surface is at about $43-44 \mathrm{~cm}$ from the plasma center. At the reversal surface $\left(r=r_{0}\right)$ the toroidal magnetic field goes to zero and has opposite sign on each side. The two lines ( solid and dotted ) in Fig. 5.5 and 5.6 represent the measured mode number spectra inside and outside of $r_{0}$, respectively.

The top plot in Fig. 5.5 is the $n$ mode number for the low-frequency case. In this case the $n$ spectra on the two sides of $r_{o}$ have their peaked values at about the same number, $n \sim 6$. This is again an indication of radially global morles because the mode number spectrum is independent of the radius. The bottom plot of Fig. 5.5 is for the high frequency case. In this case, the 


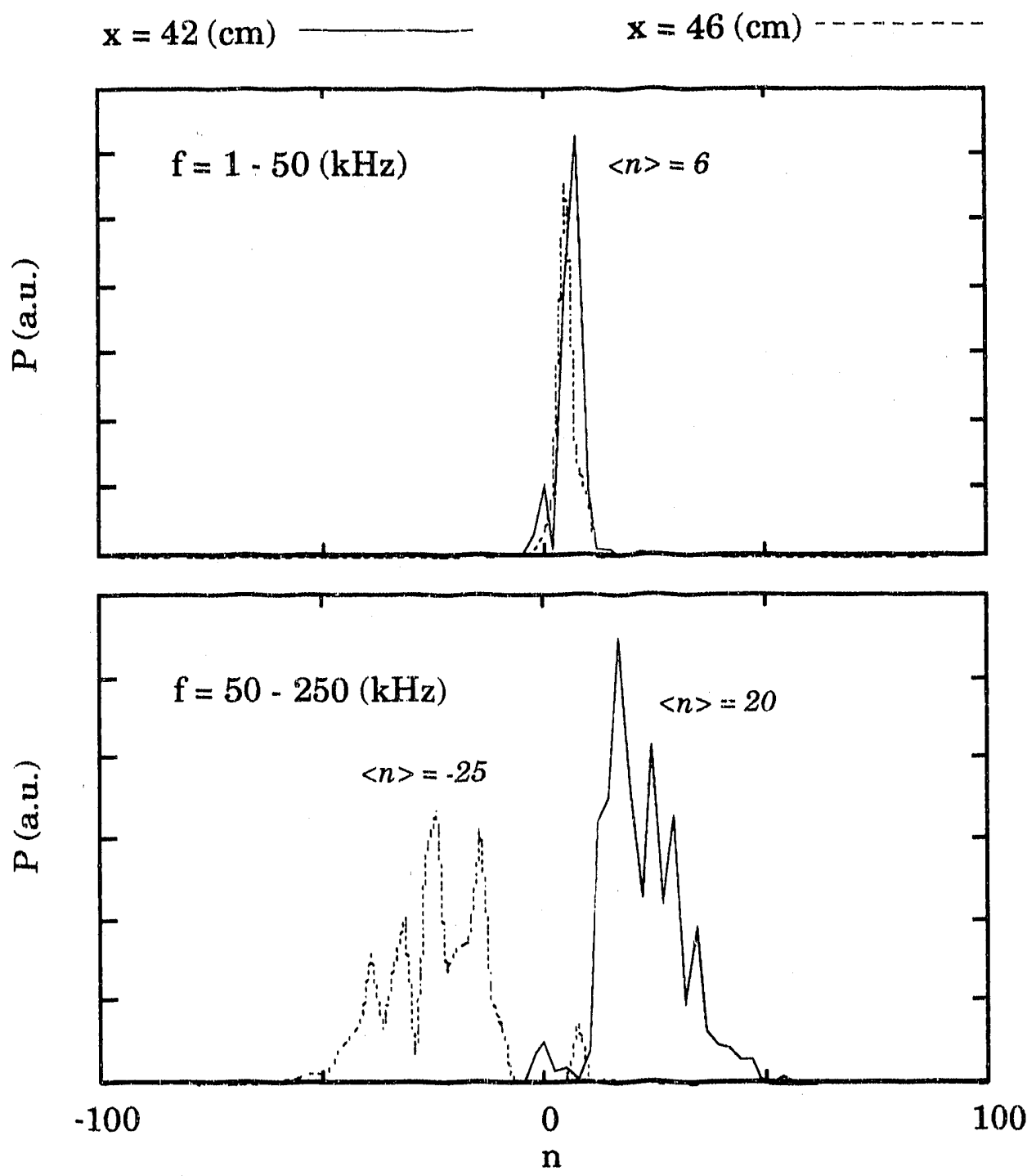

Fig. $5.5 n$ spectrum inside and outside of the reversal surface for low and high frequency magnetic fluctuations. Note that at high frequency, averaged peak n number changes sign at the reversal surface. The low freqicency part remains unchanged. 

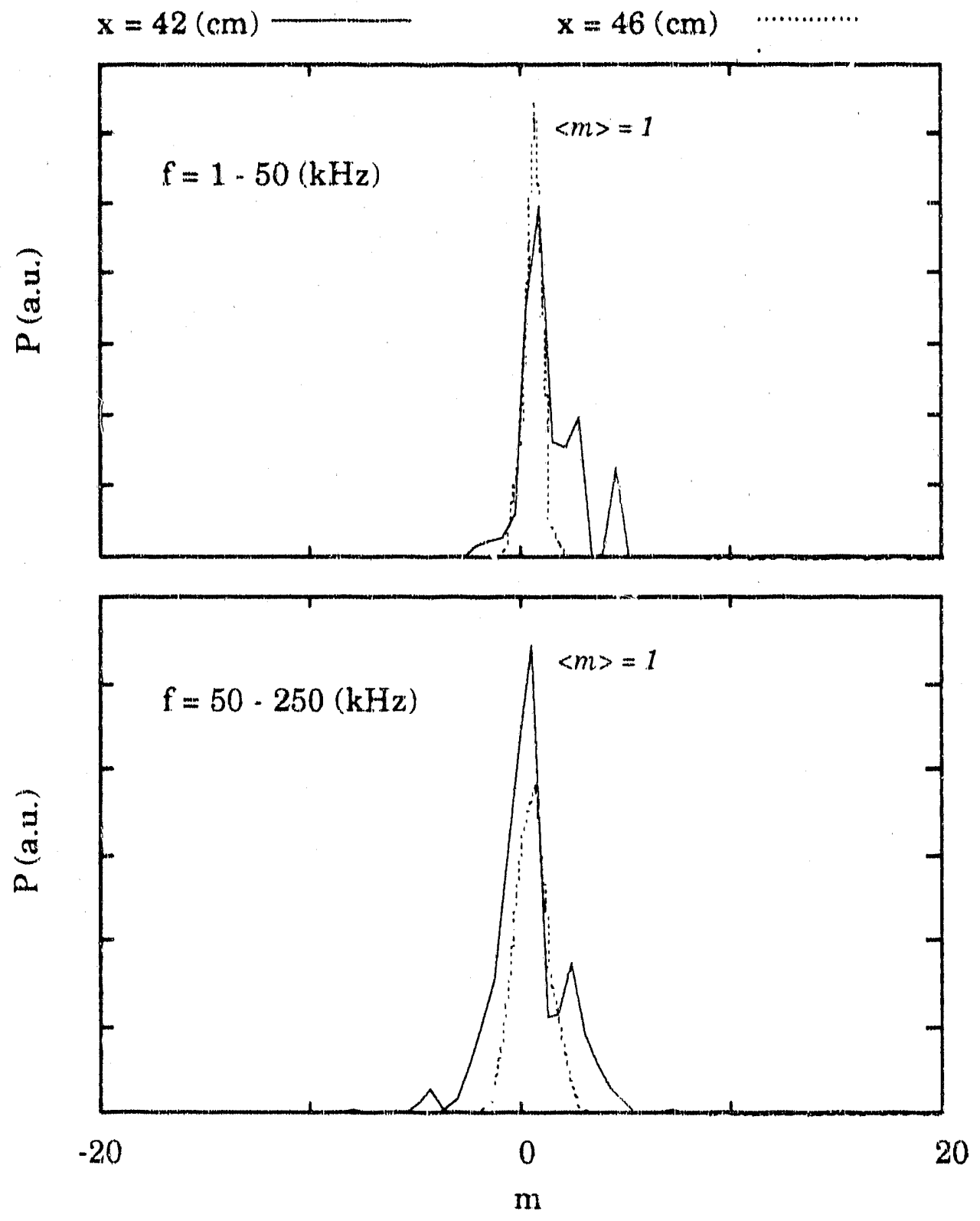

Fig. 5.6 $m$ spectrum inside and outside of the reversal surface for low and high frequency magnetic fluctuations. The spectrum is unchanged on each side of ro. There is no significant change of the spectrum with frequency. 
toroidal mode number spectra show remarkably different peak $n$ values at the two sides of $r_{0}$ The signs of the peaked $n$ values are different at two sides of $r_{0}$. At $r=46 \mathrm{~cm}$ ( outside $r_{s}$ ), $n_{\text {peak }} \sim-25$. At $r=42 \mathrm{~cm}\left(\right.$ inside $\left.r_{s}\right), n_{\text {peak }} \sim$ 20. Here, assuming $m$ (poloidal mode number) remains positive, then positive $n$ represents internally resonant modes, and negative $n$ corresponds to externally resonant modes. The assumption of positive $m$ is verified experimentally and will be described in the following.

The $m$ number spectra for those same cases are ploted in Fig. 5.6. They demonstrate that the peak values of $m$ remain at unity for both low and high freruencies. Furthermore, the peak values of $m$ are unchanged on each side of the reversal surface. The high frequency magnetic fluctuations appear to have their dominant poloidal node number independent of radial positions in the plasma. It should be noted that the $m$ spectrum becomes broader as the frequency increases.

The explanation for $n$ to change sign, but not $m$, could be as following: for $m$ to change sign ( $1 \rightarrow 0 \rightarrow-1)$, the plasma has to experience a drastic change with large scale reconnection when $m$ goes to zero. On the other hand, for $n$ to change sign $(-25 \rightarrow \infty \rightarrow 25$ ), only small scale changes would occur requiring a small amount of energy change. 
The combination of the results in Fig. 5.5 and 5.6 leads to the following important remarks: for high frequency magnetic fluctuations, the ratio of the averaged mode number $\frac{m}{n}$ tracks the value of local safety factor $q$. From discussions in the Appendix, the averaged mode number spectra, under the assumption of wave packets, could be taken as the dominant local mode number. Thus for high frequency magnetic fluctuations, the $\frac{m}{n}$ value from dominant mode number spectra matches the value of local safety factor. The mode resonant condition requires: $\frac{m}{n}=q_{(r)}$, where $q_{(r)}=\frac{r B_{t}(r)}{R B_{p}(r)}$. Fig. 5.7 is the $q$ profile calculated from MPFM4 with $F=-0.15, \Theta=1.75$. We see that $q_{(r)}$ changes its sign across the reversal surface because $B_{t}(r)$ does. Since the sign changing of $q$ value occurs only locally, the mode resonant condition is satisfied locally only. The high frequency modes are locally resonant modes. The mode number spectra for low frequency fluctuations clearly do not depend on the local $q$ value.

High frequency magnetic fluctuations have such a short radial correlation length that at each radial position in the plasma, the fluctuation is mainly influenced by the locally resonant instabilities with their resonant surfaces not more distant than their radial coherence lengths. The low frequency fluctuations, on the other hand, are the result of spatialy global modes. A 


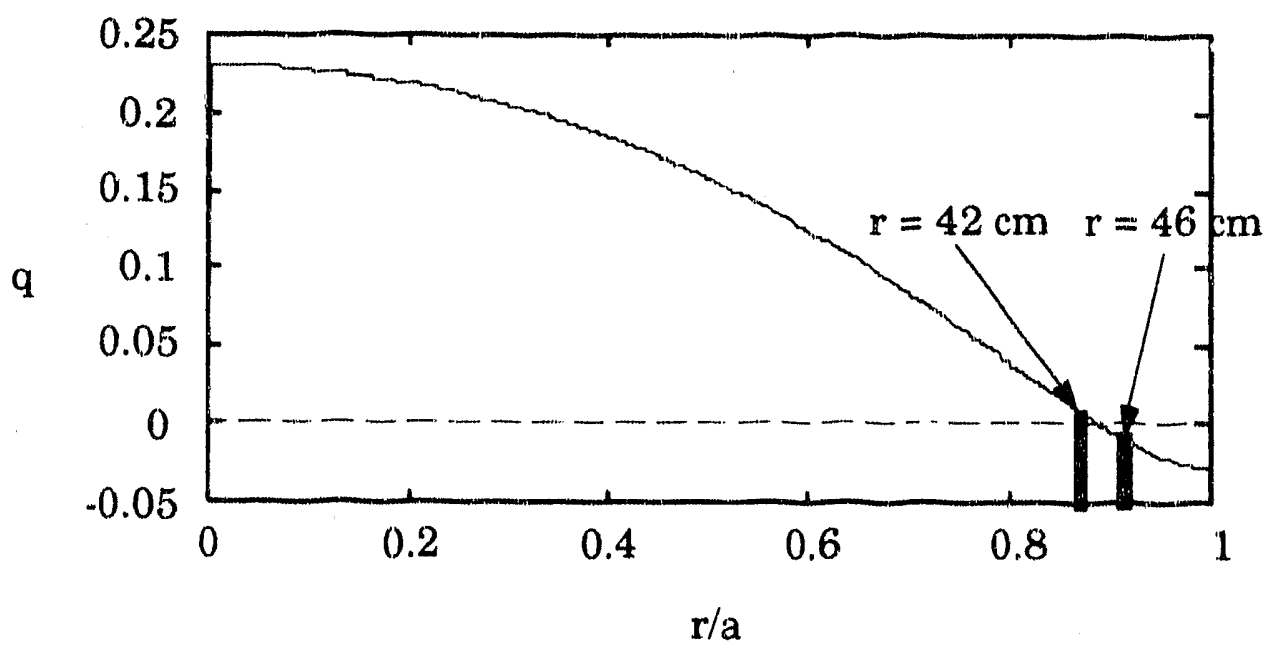

Fig. 5.7 The radial profile of $q$. This is obtained from MPFM with $F=-0.15, \Theta=1.75$.

few such modes dominate the low frequency fluctuations in the RFP plasmas.

Because of the sign change for the toroidal node number (toroidal wavenumber ) across the reversal surface $r_{0}$, the direction of the diamagnetic drift speed $v^{*}$ should also change across $r_{0}$, for high frequency magnetic fluctuations.

It should be mentioned that although the sign change of $\frac{m}{n}$ agrees with that of $q_{\text {local }}$ the exact value of the averaged $\frac{m}{n}$ may not necessarily match the value of $q_{\text {locll }}(r)$ at every $r$. The reason is as follows: Even at frequencies 
above $50 \mathrm{kHz}$, the radial correlation length is still the order of $1 \mathrm{~cm}$, as indicated in Fig. 5.4. The averaged mode number spect um at each radial position is simply the combination of neighboring resonant modes weighted by their amplitudes, rather than from a single mode resonant with $q(r)$. Since the general trend for turbulent mode spectra is that the mode amplitude decreases with increase of mode number, the measured $n$ number in general is lower than the true resonant value of $q_{\text {local }}$ at any particular $r$. Fig. 5.8 is the comparison for calculating the high frequency $n$ number spectra with different lower frequency cut-offs. It is seen ciearly that with higher low $f$ cut-off, the peak $n$ becomes higher.

The radial coherence length in Fig. 5.4 contains some important information about the characteristics of high frequency magnetic fluct'lations. By comparing it with the correlation length of electrostatic turbulences, it is possible to identify the modes that drive the turbulence. It has been indicated that ${ }^{5}$ for resistive interchange modes, the correlation length of magnetic fluctuations should have a different scale than that of the electrostatic turbulence. The correlation length of electrostatic turbulence is related to the mixing length used in the model. The microtearing instability, however, is shown ${ }^{6}$ to have magnetic and electrostatic fluctuation 

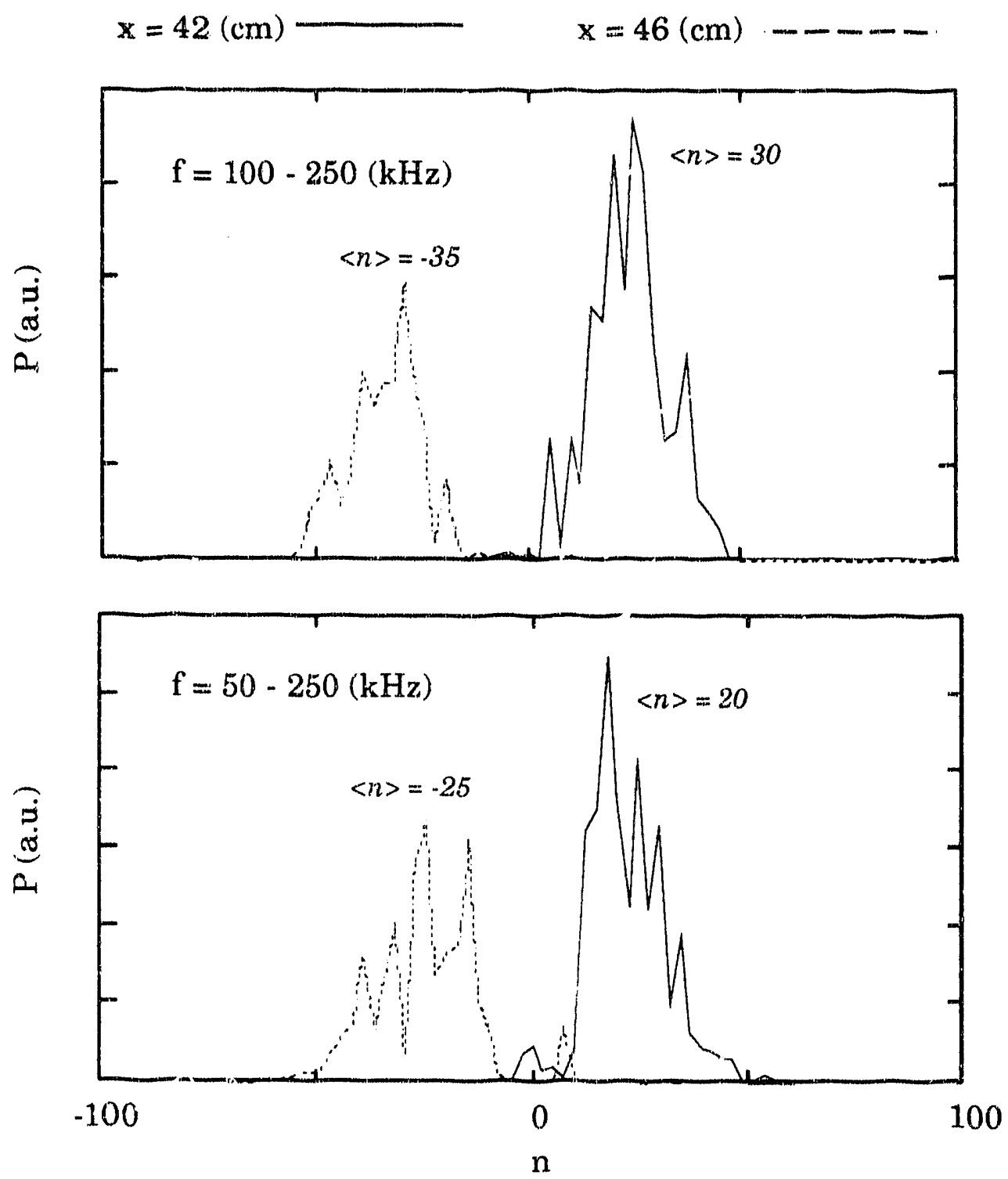

Fig. 5.7 As the lower frequency cut-off increases, the measured $n$ spectra peak at higher value, which is closer to the exact value of the resonant local safety factor. 
correlation lengths closely related.

The transverse correlation length measured from electrostatic fluctuations ${ }^{7}$ is believed somewhat shorter compared with the scale length given in Fig. 5.4. This could be taken as an indication of the occurense of resistive interchange modes in the edge of RFP on MST. The occurence of the microtearing modes described in reference 6 , on the other hand, have the same correlation lengths for both magnetic and electrostatic fluctuations. This is somewhat in contrast with the experimental result, although the radial correlation length measurement for electrostatic fluctuations is insufficient.

The conclusive identification of small scale localized magnetic fluctiations, of course, would require more accurate measurement of scale length of fluctuating quantities, and a more controllable experimental setup that allows variation of gradients of temperature and pressure profile.

The result of long $L_{r}(\omega)$ at low frequency can be directly compared with the resistive MHD theory, where the dominant $m=1$, low $n$ tearing modes have radial extent of the same order as the plasma minor radius, $a$. 


\section{REFERENCES}

1 S. Assadi, Ph. D. Thesis, University of Wisconsin-Madison, 1992.

2 E. J. Zita, Private Communication.

$3 \quad$ R. J. La Haye et. al., Physics of Fluids, 27, (1984), 2576.

4 W. Shen and J. C. Sprott, Physics of Fluids, B3, (1991), 1225.

5 B. A. Carreras and P. H. Diamond, Physics of Fluids, B1 (1989), 1011.

6 G. G. Craddock and P. W. Terry, Physics of Fluids, B3, (1991), 3286.

7 T. D. Rempel et. al., Physical Review Letters, 67, (1991), 1438. 
Chapter 6: Ambipolar Particle Flux Induced by Magnetic Fluctuations

This chapter presents the results of internal measurements of magnetic fluctuations in the RFP plasmas in MST. It is found that the particle flux induced by magnetic fluctuations at the edge of the MST is ambipolar. Interpretation of this result is given to both low and high frequency fluctuations.

\subsection{Ambipolar Particle Transport due to Magnetic Fluctuations}

As described by (2.2) and (2.3) in Chapter 2, the particle flux induced by magnetic fluctuations only are:

$$
\begin{gathered}
\Gamma_{e}=-\frac{\left\langle\tilde{j}_{e l /} \tilde{B}_{r}>\right.}{e B} \\
\Gamma_{i}=\frac{\left\langle\tilde{j}_{i / 1} \tilde{B}_{r}>\right.}{e B}
\end{gathered}
$$

The net charged particle flux, $\Gamma_{i}-\Gamma_{e}$, induced by magnetic field 
fluctuations thus can be written as:

$$
\Gamma_{n e t}=\frac{<\tilde{j}_{11} \tilde{B}_{r}>}{e B}
$$

Using the definition in the Appendix, we have for $\Gamma_{\text {net }}$

$$
\Gamma_{n e t}=\frac{\left|\tilde{j}_{\| \prime}\right|\left|\tilde{B}_{r}\right|}{e B} \gamma_{j B} \cos \left(\Delta \phi_{j B}\right)
$$

As expressed by in (6.4), the total net charged particle flux, sometimes called non-ambipolar particle flux, is proportional to the cross product of two fluctuating quantities, namely, $\tilde{j}_{/ /}$and $\widetilde{B}_{r}$. Also note that not only does the product of the amplitudes of these two quantities contribute to $\Gamma$, but also their cross-phase angle.

6.1.1 Experimental Evidence of Ambipolar Particle Flux Induced by Magnetic Fluctuations

The decomposition of $\tilde{j}_{/ /}$is:

$$
\tilde{j}_{/ \prime}=\frac{1}{B}\left(\tilde{j}_{p} B_{p}+\tilde{j}_{t} B_{t}+j_{p} \tilde{B}_{p}+j_{t} \tilde{B}_{t}-\boldsymbol{j} \cdot \hat{\boldsymbol{b}} \tilde{B}\right)
$$


Each term in (6.5) has been measured experimentally in RFP plasma on the MST. The first term is found to be the dominant one. The last 3 terms are all small compared to the first term because of the relation $\frac{\widetilde{B}}{B} \ll$ $\underset{j}{j}$. The second term in (6.5) is small at the plasma edge because in RFPs, $B_{t}$ is nearly 0 , and $B_{p}$ is finite.

Figure 6.1 shows the measured cross-coherence $\gamma_{j B}$ and cross"phase angle $\Delta \phi_{j B}$ between $\tilde{j}_{p}$ and $\widetilde{B}_{r}$. The measured coherence at low frequency is about 0.4 , and falls to about 0.15 as the frequency increases up to 250

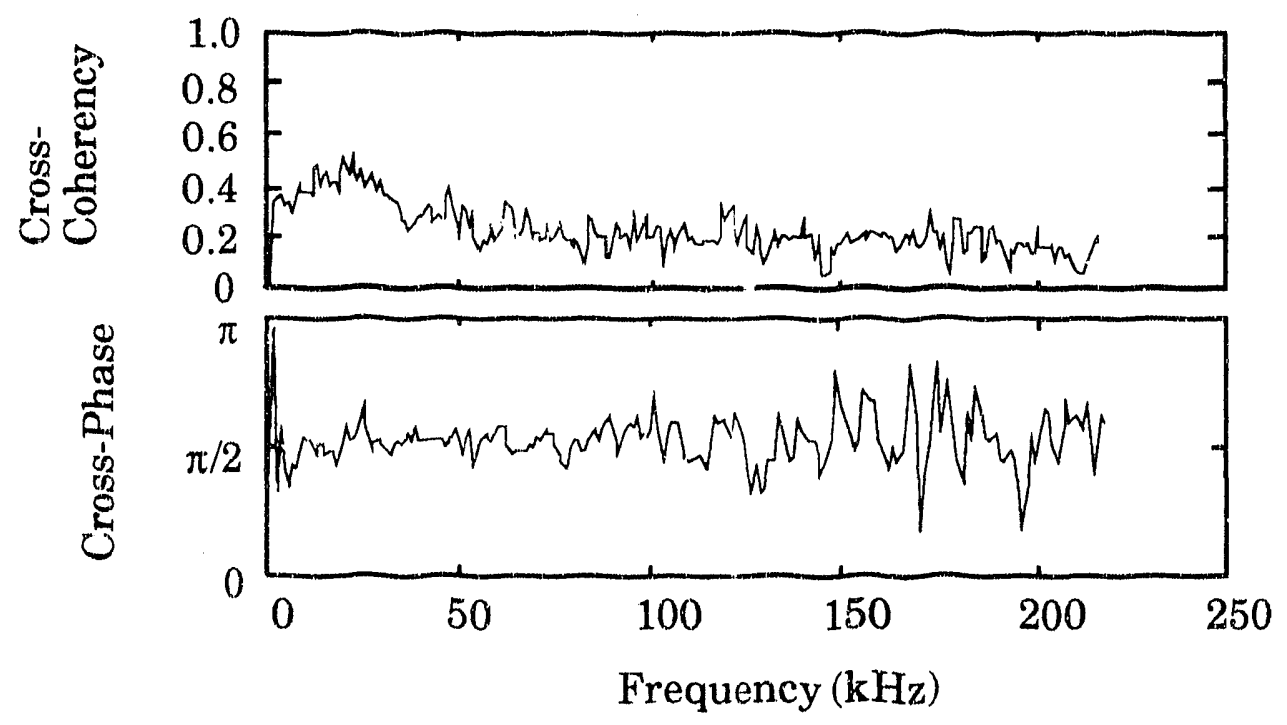

Fig. 6.1 Cross-coherence and cross-phase between measured $j_{\|}$and $B r$. The cross-phase angle is about $\pi / 2$, thus gives it non-ambiplar particle flux induced by magnetic fluctuations. 
$\mathrm{kHz}$. Experimental uncertainty in the coherence $\gamma$ is determined to be 0.04

- 0.05. The total experimental uncertainty includes the misalignment of the coils, the calibration errors of $A_{e, f}$, and in the electronincs, is about 5 - 7\%.

The cross-phase angle shown in Fig. 6.1 is close to $\frac{\pi}{2}$ throughout the frequency spectrum. This gives $\Gamma_{\text {net }} \sim 0$ because $\cos \left(\frac{\pi}{2}\right)=0$. We estimate the upper bound of $\Gamma_{n e t}$ due to experimental uncertainty. The result is: $\Gamma_{\text {upper }}$ $<2.0 \times 10^{19} \mathrm{~m}^{-2} \mathrm{~s}^{-1}$. This is less than $5 \%$ of the total particle flux for this type of discharge.

The result of zero net charged particle flux (or non-ambipolar particle flux), driven by magnetic fluctuations in RFP plasmas demonstrates that the particle transport by magnetic fluctuations is ambipolar.

The result in Fig. 5.8 is remarkable because the magnitudes of the individual quantities $j_{/ /}$and $B_{r}$ are large. Had the phase angle not been close to $\frac{\pi}{2}, \Gamma_{\text {net }}$ would have been large, meaning plasmas are losing charge (negative charge) at a large rate unless some other loss mechanisms are present.

\subsection{Interpretation of Ambipolar Flux Induced by Magnetic} Fluctuations 
In the low frequency range the ambipolarity of particle flux due to magnetic fluctuations can be interpreted as the necessary condition sei by the phise relations among components for tearing modes. From Ampere's law:

$$
\mu_{0} \tilde{j}_{p}=\frac{\partial \tilde{B}_{t}}{\partial r} \cdot \frac{1}{R}\left(\frac{\partial \tilde{B}_{r}}{\partial \phi}\right)
$$

In section 5.1, the low frequency $m=1$ MHD modes are identified as having the structure of radial standing and toroidally propagating perturbation waves. Upon Fourier decomposition, the propagation can be represented by a factor $e^{-(k \cdot r \cdot \omega *)}$ multiplying each of the Fourier components. Thus the second term in $(5.6)$ is out of phase $\left(\Delta \phi=\frac{\pi}{2}\right)$ with $B_{r}$. by the factor $i k_{t}$. The first term, by the definition of radial standing waves, is in phase with $B_{t}$. Fig. 5.3 demonstrates that $\Delta \phi$ between $B_{t}$ and $B_{r}$ is $\frac{\pi}{2}$, Thus $\Delta \phi$ between $j_{/ /}$and $B_{r}$ is also $\frac{\pi}{2}$.

The ambipolarity at high frequency can be somewhat explained using the simple argument of Waltz ${ }^{2}$. As described in 2.3.2, he considers localized modes for which the fluctuating magnetic field is transverse to the 
equilibrium magnetic field $\left(B_{/ /} \sim 0\right)$. These conditions are well satisfied for the measured fluctuations for which $\widetilde{B}_{/ /} \ll \widetilde{B}_{\perp}$ and $\frac{\widetilde{B}_{\|}}{B} \ll 10^{-4}$. In this case, a consequer.ce of Ampere's law is that $\left\langle\tilde{j}_{\|} \tilde{B}_{r}>\right.$ vanishes when integrated over the small radial exterst of the mode. 


\section{REFERENCES}

$1 \quad$ R. E. Waltz, Physics of Fluids, 25, (1982), 1269. 


\section{Chapter 7 Summary and Conclusions}

We have successfuhly moasured ${ }^{1}$ plasma current density and current density fluctuations using the "forked" probe with multiple magnetic sensing coils. The measured $j$ and $\tilde{j}$ agree with the predictions of resistive MHD simulations.

We also have measured toroidal and poloidal mode number spectra using the same probe. We have found: 1). The high frequency magnetic fluctuations are localized and locally resonant modes. This is evidenced by the sensitive dependence of wavenumber (mode number) spectrum on radial locations in the plasma; 2). Low frequency fluctuations are consistent with the global teaxing instabilities predicied by MHD codes.

Based on the measurement of $\widetilde{B}$ and $\tilde{j}$, we are able to obtain the nonambipolar part of the particle flux induced by magnetic fluctuations. This is done by calculating the cross-product of $\left\langle\widetilde{j}_{\|} \widetilde{B}_{r}\right\rangle$. It is found that this nonambipolar flux is zero for fluctuation frequencies between $1 \mathrm{kHz}$ and 250 $\mathrm{kHz}$. The particle flux due to magnetic fluctuations is thus proved to be ambipolar. 


\section{REFERENCFS}

1. W. Shen et. al., Physical Review Letters, 68, (1992), 1319. 


\section{Appendix}

The purpose of this appendix is to present some detailed algorithms for spectral analysis.

The problem of analyzing fluctuation data associated with turbulence has developed tremendously over the past decade. The most widely used method is the Fast-Fourier-Transform (FFT) spectral analysis. It can give much fundamental imformation about the fluctuations themselves, and about the relationship among several fluctuation signals.

a). Power Spectra, Cross-cohernece and Cross Phase

Let $g_{1(t)}$ and $g_{2(t)}$ denote two fluctuating signals, one could first transform the data to frequency spectra $G_{1(f)}$ and $G_{2} f$ via the FFT algorithm. The resultant cross-power spectrum is computed from

$$
\left.<P_{12(f)}\right\rangle=\left\langle G_{1(f)}^{*} G_{2(f)}>\right.
$$

where * denotes a complex conjugate, and $<>$ denotes an ensemble average. 
Since $P_{12(f)}$ is in general a complex number, it may be expressed as:

$$
P_{12(f)}=\left|P_{12(f)}\right| \exp \left[i \theta_{12(f)}\right]
$$

with $\left|P_{12(f)}\right|$ the cross-amplitude spectrum given by

$$
<\left|P_{12(f)}\right|>=<\left|G_{1(f)}\right|><\left|G_{g(f)}\right|>
$$

and $\theta_{12(f)}$ the phase spectrum given by

$$
\theta_{12(f)}=\operatorname{atan}^{-1}\left\{\frac{\operatorname{Im}\left[P_{12(f)}\right]}{\operatorname{Re}\left[P_{12(f)}\right]}\right\}
$$

The auto-power spectra are given by

$$
\begin{aligned}
& \left\langle P_{11(f)}\right\rangle=\left\langle\left|G_{1(f)}\right|^{2}\right\rangle \\
& \left\langle P_{22(f)}\right\rangle=\left\langle\left|G_{2(f)}\right|^{2}\right\rangle
\end{aligned}
$$

and they are real and positive. The auto-power spectrum is the measure of a 
signal amplitude on a per unit-bandwidth basis.

The cross-coherence spectrum is defned as the normalized cross-power spectrum as follows:

$$
\left\langle\gamma_{12(f)}\right\rangle=\frac{\left\langle P_{12(f)}\right\rangle}{\left[\left\langle P_{1(f)}\right\rangle\left\langle P_{2(f)}\right\rangle\right]^{1 / 2}}
$$

We see that $\left|\gamma_{12(f)}\right| \leq 1$ for all $f$. If $\left|\gamma_{12(f)}\right|$ is unity, then we say that signal 1 and signal 2 are perfectly coherent. If $\left|\gamma_{12(f)}\right|$ is 0 , we say they are incoherent. If . $\left|\gamma_{12(f)}\right|<1$, we say they are partially coherent.

From the cross-phase spectra one could obtain a averaged dispersion relation, assuming there is one. The dispersion relation is given by:

$$
k_{(f)}=\frac{\theta_{12(f)}}{\Delta x}
$$

and $\Delta x$ is the spatial distance between point 1 and point 2 at which the signals $g_{1(t)}$ and $g_{2(t)}$ are measured.

Because the real experimental signals are in general stochastic and turbulent, only the ensemble averages of $P_{12(f)}, \gamma_{12(f)}$ and $\theta_{12(f)}$ are meaningful. The number of terms used for ensemble averages determines the 
statistical uncertainties when calculating power spectra, cross-coherence and cross phase.

b).Wavenumber and Frequency Spectra

The spectral analysis method discussed in a) encounters severe limitations. First, it gives only the spectra in the frequency domain whereas most of the theoretical results are given in the wavenumber( $k)$ domain. Second, the true turbulence in genaral has no deterministic dispersion relation between frequency $\omega$ and wavenumber $k$.

From two-point correlation study, one could estimate the wavenumber and frequency spectra assuming the turbulence satisfies wave packet conditions. ${ }^{1}$ This method calculates the so-called local wave number spectrum and approximates it to the true wavenumber spectrum of the turbulence.

As discussed in a), the power and average wavenumber spectra can be calculated via FFT. The local wavenumber and frequency spectra can thus be computed by summing the sample power values $S_{(\omega)}$ at a fixed frequency $\omega$ from those records which have a sample wavenumber in the range of $K$ to 
$K+\Delta K$

$$
\left.S_{l}(k, \omega)=\frac{1}{M} \sum_{j=1}^{M} I_{[0, \Delta K]}\left[k-k_{j}^{j}(\omega)\right)\right] \times \frac{1}{2}\left[P_{l}^{j}(\omega)+P \dot{2}_{2}(\omega)\right]
$$

with the indicator function $I_{[0, h]}(x)$ defined as

$$
\begin{aligned}
I_{[0, h]}(x) & =1, \text { when } 0 \leq x h \text { and } \\
& =0, \text { elsewhere. }
\end{aligned}
$$

$S_{l}(k, \omega)$ is called the local frequency-wavenumber spectrum. When the wave-packet assumptions in Ref. 1 are satisfied, $S_{l}(k, \omega)$ yields an estimate of the convetional spectral density $S(k, \omega)$. From this estimation, one gets the power spectra in the wavenumber domain:

$$
S(k)=\int d \omega S(k, \omega)
$$

or the estimation for the mode number spectra providing $n=k_{\phi} R$ or $m=k_{\theta} a$.

$S(k, \omega)$ is one of the most fundamental quantities describing space-time varying fluctuations. From this quantity, many of the important spectral 
quantities, such as the statistical disperssion relation and wavenumber spectrum width can thus be deduced. 


\section{REFERENCES}

1 J. M. Beall et. al., Journal of Applied Physics, 53, (1982), 3933. 

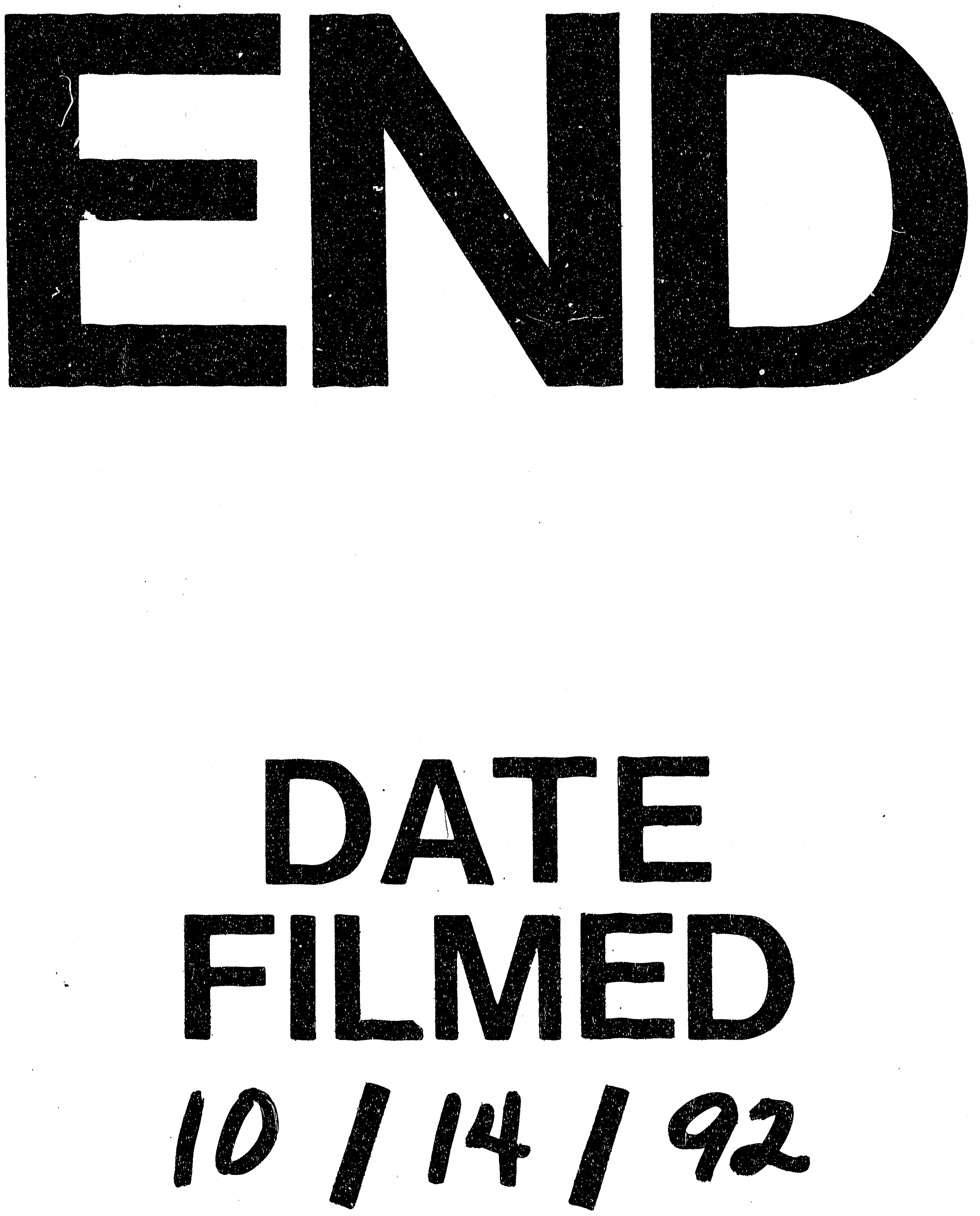
